\title{
Pacific
}

Journal of

Mathematics

\section{ON 4-MANIFOLDS, FOLDS AND CUSPS}

STEFAN BEHRENS 


\title{
ON 4-MANIFOLDS, FOLDS AND CUSPS
}

\author{
STEFAN BEHRENS
}

\begin{abstract}
We study simple wrinkled fibrations, a variation of the simplified purely wrinkled fibrations of Williams (Geom. Topol. 14:2 (2010), 1015-1061), and their combinatorial description in terms of surface diagrams. We show that simple wrinkled fibrations induce handle decompositions of their total spaces which are very similar to those obtained from Lefschetz fibrations. The handle decompositions turn out to be closely related to surface diagrams and we use this relationship to interpret some well known operations on 4-manifolds in terms of surface diagrams. This, in turn, allows us classify all closed 4-manifolds which admit simple wrinkled fibrations of genus one, the lowest possible fiber genus.
\end{abstract}

\section{Introduction}

After the pioneering work of Donaldson [1999] and Gompf [1999] on symplectic 4-manifolds and Lefschetz fibrations and of Auroux, Donaldson and Katzarkov on near-symplectic 4-manifolds [Auroux et al. 2005], the study of singular fibration structures on smooth 4-manifolds has received considerable attention in the research literature. Among the highlights in the field have been existence results for so called broken Lefschetz fibrations over the 2-sphere on all closed, oriented 4-manifolds [Akbulut and Karakurt 2008; Baykur 2008; Gay and Kirby 2007; Lekili 2009] as well as a classification of these maps up to homotopy [Lekili 2009; Williams 2010]. Furthermore, the classical observation that Lefschetz fibrations over the 2-sphere are accessible via handlebody theory and can be described more or less combinatorially in terms of collections of simple closed curves on a regular fiber known as the vanishing cycles [Kas 1980; Gompf and Stipsicz 1999] was extended to the broken Lefschetz setting in [Baykur 2009].

Our starting point is the work of Williams [2010], who introduced the closely related notion of simplified purely wrinkled fibrations, proved their existence and exhibited a similar combinatorial description of these maps - again by collections of simple closed curves on a regular fiber - which he calls surface diagrams. It

MSC2010: primary 57M50; secondary 57R65.

Keywords: 4-manifolds, folds, cusps, simple wrinkled fibrations, simplified purely wrinkled

fibrations, broken Lefschetz fibrations, surface diagram. 


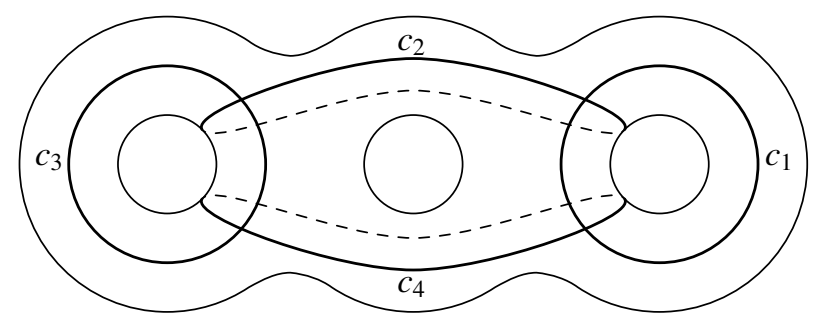

Figure 1. A surface diagram of $S^{1} \times S^{3} \# S^{1} \times S^{3}$ due to Hayano [2012].

follows that all smooth, closed, oriented 4-manifolds can be described by surface diagrams; an example of such a diagram is shown in Figure 1. However, the correspondence between simplified purely wrinkled fibrations and surface diagrams has been somewhat unsatisfactory in that it usually involved arguments using broken Lefschetz fibrations and the assumption that the fiber genus is sufficiently high.

It is one of our goals to provide a detailed and intrinsic account of this correspondence and to clarify the situation in the lower-genus cases. Once this is done we give some applications.

We now describe the contents of this paper in more detail. In Section 2 we begin by recalling some preliminaries from the singularity theory of smooth maps and the theory of mapping class groups of surfaces. This section is slightly lengthy because we intend to use it as a reference for future work.

The following two sections form the technical core of this paper. In Section 3 we introduce simple wrinkled fibrations over a general base surface; in the case when the base is the 2-sphere our definition is almost equivalent to Williams' simplified purely wrinkled fibrations and our reason for introducing a new name is mainly to reduce the number of syllables. We explain how the study of simple wrinkled fibrations reduces to certain fibrations over the annulus which we call annular simple wrinkled fibrations to which we associate twisted surface diagrams; roughly, such a diagram consists of a closed, oriented surface $\Sigma$, an ordered collection of simple closed curves $c_{1}, \ldots, c_{l} \subset \Sigma$ and an orientation-preserving diffeomorphism $\mu: \Sigma \rightarrow \Sigma$ such that pairs of consecutive curves $\left(c_{i}\right.$ and $c_{i+1}$ for $i<l$, as well as $\mu\left(c_{l}\right)$ and $c_{1}$ ) intersect transversely in one point. We prove the following:

Theorem 1.1. There is a bijective correspondence between annular simple wrinkled fibrations up to equivalence and twisted surface diagrams up to equivalence.

For precise definitions we refer to Section 3. In the course of the proof we show that annular simple wrinkled fibrations induce (relative) handle decompositions of their total spaces which are, in fact, encoded in a twisted surface diagram (Section 3B). These handle decompositions bear a very close resemblance with those obtained from Lefschetz fibrations; the only difference appears in the framings 
of certain 2-handles. The section ends with an investigation of the ambiguities for gluing surface bundles to the boundary components of annular simple wrinkled fibrations.

In Section 4 we specialize to the case when the base surface is either a disk or a sphere and recover Williams' setting. Using our results about annular simple wrinkled fibrations we obtain a precise correspondence between Williams' (untwisted) surface diagrams and simple wrinkled fibrations over the disk (Proposition 4.1) and the sphere (Corollary 4.2). In particular, our approach provides a direct way to construct a simple wrinkled fibration from a given surface diagram circumventing the previously necessary detour via broken Lefschetz fibrations. ${ }^{1}$

Next, we address the subtle question of which surface diagrams give rise to simple wrinkled fibrations over the sphere and thus describe closed 4-manifolds. Just as in the theory of Lefschetz fibrations, the key is to understand the boundary of the associated simple wrinkled fibration over the disk. We show how to identify this boundary with a mapping torus and describe its monodromy in terms of the surface diagram. Unfortunately, it turns out that the boundary is much harder to understand than in the Lefschetz setting.

We then go on to review the handle decompositions exhibited in Section 3 when the base is the disk or the sphere and describe a recipe for drawing Kirby diagrams for them. To complete the picture, we compare our decompositions with the ones obtained via simplified broken Lefschetz fibrations.

In Sections 5 and 6 we give some applications. We show that certain substitutions of curve configurations in surface diagrams correspond to cut-and-paste operations on 4-manifolds. In particular, we give a surface diagram interpretation of blowups and sum stabilizations, by which we mean connected sums with $\mathbb{C} P^{2}, \overline{\mathbb{C} P^{2}}$ and $S^{2} \times S^{2}$. Using these we easily obtain a classification of closed 4-manifolds which admit simple wrinkled fibrations with the lowest possible fiber genus.

Theorem 1.2. A smooth, closed, oriented 4-manifold admits a simple wrinkled fibration of genus one if and only if it is diffeomorphic to $k S^{2} \times S^{2}$ or $m \mathbb{C} P^{2} \# n \overline{\mathbb{C} P^{2}}$ where $k, m, n \geq 1$.

Our result should be compared to [Baykur and Kamada 2010] and [Hayano 2011], where the classification problem of genus-one simplified broken Lefschetz fibrations is addressed but only partial solutions are achieved. However, it should also be noted that their class of maps is strictly larger than that of genus-one simple wrinkled fibrations and it is thus conceivable that the classification is more complicated.

Section 7 closes this paper by highlighting what we consider as some of the main problems in the field and by outlining some related developments.

\footnotetext{
${ }^{1}$ By now this can be considered as a special case of [Gay and Kirby 2012], which appeared while we were writing this paper.
} 
Conventions. By default, all manifolds are smooth, compact and orientable; all maps are smooth and all diffeomorphisms preserve orientations. Given a submanifold $S \subset M$ we denote by $v S$ (respectively $\bar{v} S$ ) an open (respectively closed) tubular neighborhood of $S$ and whenever we speak of neighborhoods of submanifolds we usually mean tubular neighborhoods. For induced orientations on boundaries we use the outward normal first convention and, in order to coherently orient regular fibers of maps between oriented manifolds, we use the fiber first convention. Exceptions to these rules will be explicitly stated and we reserve the right to sometimes restate some of the conditions for emphasis.

\section{Preliminaries}

To fix some terminology, let $f: M \rightarrow N$ be a smooth map with differential $d f: T M \rightarrow T N$. A critical point or singularity of $f$ is a point $p \in M$ such that $d f_{p}$ is not surjective. The set of critical points, called the critical locus of $f$, will be denoted by

$$
\mathcal{C}_{f}:=\left\{p \in M \mid \operatorname{rk} d f_{p}<\operatorname{dim} N\right\} \subset M .
$$

The image of a critical point is called a critical value and the set of all critical values is called the critical image of $f$.

As customary, we call the preimage of a point a fiber, usually decorated with the adjectives regular or singular indicating whether or not the fiber contains singularities. Note that regular fibers are always smooth submanifolds with trivial normal bundle.

Remark 2.1. The terms critical point and singularity are used synonymously and somewhat inconsistently in the literature, even in standard references such as [Golubitsky and Guillemin 1973]. We will adapt to this custom of arbitrariness and also use both terms depending on which seems more appropriate. However, we would like to stress that neither term indicates the failure of a map to be smooth at a given point — all maps we consider are smooth — they just indicate irregular behavior of the differential at that point as described above.

2A. Folds, cusps and Lefschetz singularities. As a warm-up, recall that a generic map from any compact manifold to a 1-dimensional manifold has only finitely many critical points on which it is injective and, moreover, all critical points are of Morse type; that is, they are locally modeled on maps of the form

$$
\left(x_{1}, \ldots, x_{n}\right) \mapsto-x_{1}^{2}-\cdots-x_{k}^{2}+x_{k+1}^{2}+\cdots+x_{n}^{2},
$$

where the number $k$ is called the (Morse) index of the critical point. (We say that a map $f: M^{m} \rightarrow N^{n}$ is locally modeled around $p \in M$ on $f_{0}: \mathbb{R}^{m} \rightarrow \mathbb{R}^{n}$ if there are local coordinates around $p$ and $f(p)$ mapping these points to the origin such that 
the coordinate representation of $f$ agrees with $f_{0}$.) Maps whose critical points are all of Morse type are called Morse functions.

A similar statement holds for maps to surfaces. For convenience we take the source to be 4-dimensional from now on. In this setting the Morse critical points are replaced by two other types of singularities known as folds and cusps which can also be described in terms of local models. The model for a fold point is the map $\mathbb{R}^{4} \rightarrow \mathbb{R}^{2}$ given by the formula

$$
(t, x, y, z) \mapsto\left(t,-x^{2}-y^{2} \pm z^{2}\right)
$$

and the cusps are locally modeled on

$$
(t, x, y, z) \mapsto\left(t,-x^{3}+3 t x-y^{2} \pm z^{2}\right) .
$$

If the sign in either of the above expressions is positive (respectively negative), then the singularity is called indefinite (respectively definite).

An easy calculation shows that the critical loci of the fold and cusp models are given by $\{(r, 0,0,0) \mid r \in \mathbb{R}\}$ and $\left\{\left(r^{2}, r, 0,0\right) \mid r \in \mathbb{R}\right\}$, respectively. As a consequence, the critical image of a smooth map is a smooth 1-dimensional submanifold near fold and cusp points. The critical images of both models are shown in Figure 2. Note that the critical image is smoothly embedded in the fold model whereas in the cusp case it is topologically embedded via a smooth homeomorphism whose inverse fails to be smooth only at the cusp point.

It follows directly from the models that folds always come in 1-dimensional families on which the map restricts to an immersion. We will usually be sloppy and refer to such an arc of fold points in the source as well as their image in the target as fold arcs. Furthermore, cusps are isolated in the critical locus in the sense that there is a small neighborhood which contains no other cusps. However, cusps are not isolated singularities. In fact, one can show that any cusp is surrounded by two fold arcs, at least one of which is indefinite.

We can now state the normal form of generic maps from 4-manifolds to surfaces.
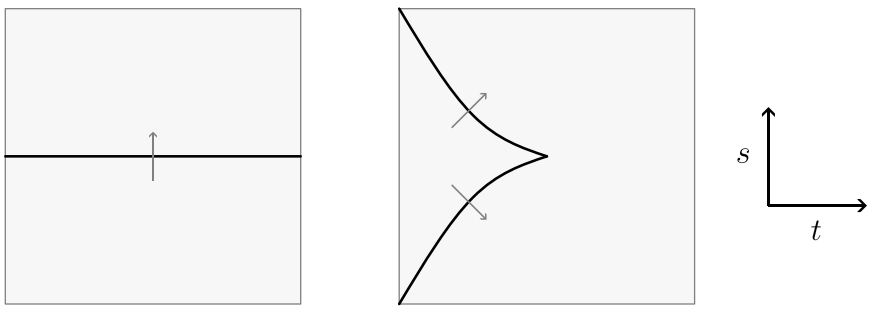

Figure 2. The critical images of the fold and cusp models. 
Theorem 2.2 (generic maps to surfaces). A generic smooth map from a 4-manifold to a surface has only fold and cusp singularities, it is injective on the cusps and restricts to an immersion of its critical locus with only transverse intersections between fold arcs.

Results of this kind are common knowledge in singularity theory; precise references for Theorem 2.2 are [Golubitsky and Guillemin 1973, Theorem 5.2] and [Levine 1964, Theorem 1] (see also [Boardman 1967; Morin 1965]).

The preceding discussion shows, in particular, that the critical locus of a generic map to a surface is a smooth 1-dimensional submanifold of the source.

Remark 2.3. Recently, these generic maps to surfaces have appeared under the name Morse 2-functions in [Gay and Kirby 2011a; 2011b; 2012].

In what follows we only deal with indefinite singularities. So from now on, when we speak of folds and cusps, we always mean the indefinite ones.

Figure 2 contains some further decorations which we will now explain. Both folds and cusps are intimately related to 3-dimensional Morse-Cerf theory. The fold models a trivial homotopy of a Morse functions with one critical point (of index two) on the vertical slices. This means that the model restricted to a small arc transverse to the fold locus is a Morse function with one critical point of index one or two, depending on the direction. The arrows in the picture indicate the direction in which the index is two. Note that the topology of the fibers of either side of a fold arc is necessarily different.

Similarly, the cusp is also a homotopy of Morse functions on the vertical slices, although a nontrivial one. It models the cancellation of a pair of critical points of index one and two. The arrows indicate the index two direction of the fold arcs adjacent to the cusp.

For the moment, this is all we have to say about folds and cusps. Another important type of singularity which has its roots in (complex) algebraic geometry is the Lefschetz singularity and its local model is given in complex coordinates by

$$
L: \mathbb{C}^{2} \rightarrow \mathbb{C}, \quad(z, w) \mapsto z w .
$$

At this point it becomes important whether the charts that we use to model the map are orientation-preserving. Indeed, the use of orientation-reversing charts for the Lefschetz model produces so called achiral Lefschetz singularities which are not compatible with complex geometry; in orientation-preserving coordinates achiral Lefschetz singularities can be modeled by $(z, w) \mapsto \bar{z} w$ which is not holomorphic. We will thus always use orientation-preserving charts to model singularities whenever the source or target are oriented. Note that this is no restriction for folds and cusps since both models admit an orientation-reversing diffeomorphism which leaves the map invariant. 
As stated in the introduction, maps with (indefinite) fold, cusp and Lefschetz singularities have been prominently featured in the research literature over the past decade. Unfortunately, different authors have used different names for various types of maps and there is yet no commonly accepted terminology in the field. For the purpose of this paper we use this:

Definition 2.4. Let $f: X \rightarrow B$ be a surjective map from an oriented 4-manifold to an oriented surface, with critical locus $\mathcal{C}_{f}$. Assume that all intersections in the critical image are transverse intersections of fold arcs and $\mathcal{C}_{f}$ is transverse to the boundary of $X$. We call

(a) a wrinkled fibration if $\mathcal{C}_{f}$ contains only indefinite folds and cusps,

(b) a (broken) Lefschetz fibration if $\mathcal{C}_{f}$ contains only Lefschetz singularities (and indefinite folds),

(c) a broken fibration if $\mathcal{C}_{f}$ contains only indefinite folds, cusps and Lefschetz singularities.

We will usually refer to $X$ as the total space and to $B$ as the base of $f$.

If $f: X \rightarrow B$ is a broken fibration, then $\partial X \cap \mathcal{C}_{f}$ is either empty or consists of finitely many fold points and it follows from the fold model that $f$ restricts to a circle valued Morse function over each boundary component of $B$.

The regular fibers of $f$ are orientable surfaces and our conventions determine an orientation. We will usually assume that $\partial X=f^{-1}(\partial B)$ so that the fibers are closed surfaces.

It is quite useful to think of broken fibrations as singular families of surfaces parametrized by the base. More precisely, the images of the folds and cusps cut the base into several regions which may or may not contain Lefschetz singularities. Each regular fiber is an orientable surface whose topological type depends only on the region that it maps into. One thus decorates the base with the topological type of the fibers over each region together with some information about what happens to a fiber if one crosses a fold arc (the fold vanishing cycles corresponding to the little arrows we have indicated above, see Definition 3.11) or runs into a Lefschetz singularity (the Lefschetz vanishing cycle). Under certain circumstances this data is enough to determine the map as we will see later on; see also [Gay and Kirby 2012].

We finish this section with a short review of the homotopy classification of broken fibrations over $S^{2}$ that was mentioned in the introduction. An important contribution of Lekili [2009] is that he showed how to pass back and forth between broken Lefschetz fibrations and wrinkled fibrations via two local homotopies, i.e., homotopies supported in arbitrarily small balls. As portrayed in Figure 3 one can wrinkle a Lefschetz point into an indefinite triangle (that is, an indefinite circle with 

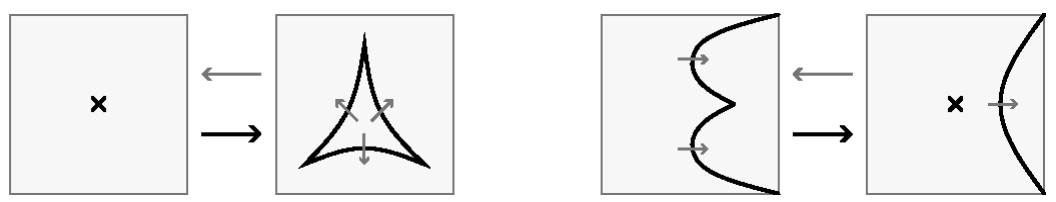

Figure 3. Wrinkling (left) and unsinking (right) a Lefschetz singularity.

three cusps) and one can exchange a cusp for a Lefschetz singularity; this move is sometimes called unsinking a Lefschetz point from a fold. (Moreover, he showed that these modifications work equally well with achiral Lefschetz singularities which, together with the results of [Gay and Kirby 2007], proves the existence of broken Lefschetz fibrations.) As a consequence, one can translate questions about broken fibrations into questions about wrinkled fibrations which are accessible by means of singularity theory. For example, there is a structural result similar to Theorem 2.2 for generic homotopies between wrinkled fibrations. The basic building blocks include isotopies of the base and total space and three types of modifications (and their inverses) that are realized by local homotopies: the birth/death, the merge and the flip. Figure 4 shows their effect on the critical image. In general, such a generic homotopy will pass through maps with definite singularities. However, the main theorem in [Williams 2010], which was conjectured in [Lekili 2009], states that indefinite singularities can, in fact, be avoided. In other words, any two homotopic wrinkled fibrations are homotopic through wrinkled fibrations.

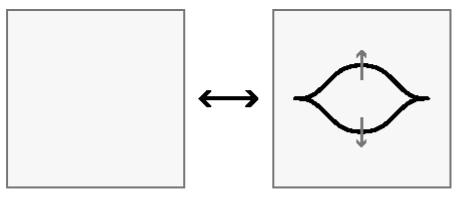

birth

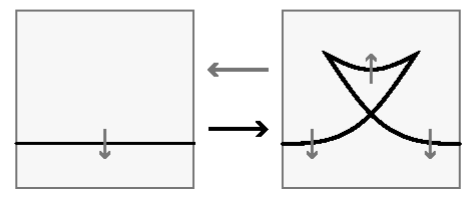

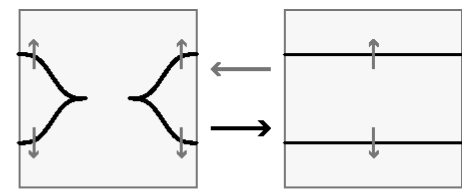

merge

flip

Figure 4. The basic local homotopies.

Remark 2.5. It has become common to refer to an application of any of these modifications as moves performed on a broken fibration; this terminology is due to [Lekili 2009]. It is important to note that most of these moves are not strictly reversible in the following sense. If the critical image of a given broken fibration exhibits the left configuration in any of the pairs, it is always possible to replace it by the one on the right. However, it might not be possible to go into the other 
direction. The only exception is the birth. In all other cases some extra conditions are needed to go from right to left. This is indicated in our pictures with shaded arrows. For further details we refer to [Lekili 2009].

Remark 2.6. There is some disagreement in the literature about which direction in the second pair in Figure 4 should be called merge and which inverse merge. To avoid this decision we simply speak of merging cusps and merging folds, respectively.

2B. Surfaces and simple closed curves. As we pointed out, the regular fibers of broken fibrations are surfaces and these fibers will be our main focus later on. The theory of surfaces and mapping class groups is yet another field of mathematics with many different conventions and, in the author's experience, it can be confusing to decide whether a statement in some reference actually applies to a situation at hand. For this reason we give very precise definitions, deliberately risking to be overly precise.

By a surface $\Sigma$ we mean a compact, orientable, 2-dimensional manifold, possibly with boundary and some marked points in the interior. A simple closed curve in $\Sigma$ is a closed, connected, 1-dimensional submanifold of $\Sigma$ that does not meet the boundary or the marked points. We usually consider simple closed curves up to ambient isotopy in $\Sigma$ relative to $\partial \Sigma$ and the marked points and will not make a notational distinction between a simple closed curve and its isotopy class. Note that according our definition simple closed curves are unoriented objects. However, from time to time it will be convenient to choose orientations on them in order to speak of their homology classes.

The geometric intersection number of two simple closed curves $a, b \subset \Sigma$ is

$$
i(a, b):=\min \{\#(\alpha \cap \beta) \mid \alpha \sim a, \beta \sim b, \alpha \pitchfork \beta\} \in \mathbb{N},
$$

where the signs $\sim$ and $\pitchfork$ indicate isotopy and transverse intersection. If the curves as well as the surface are oriented, then we also have an algebraic intersection number which is obtained by a signed count of intersections after making the curves transverse. Equivalently, this number can be described as

$$
\langle a, b\rangle:=\langle[a],[b]\rangle_{\Sigma}:=\langle[a],[b]\rangle_{H_{1}(\Sigma)} \in \mathbb{Z},
$$

where the bracket on the right side denotes the intersection form on $H_{1}(\Sigma)$. (In the present paper homology is always taken with integer coefficients.)

Note that the algebraic intersection number is alternating and depends only on the homology classes of the oriented simple closed curves while the geometric intersection number is symmetric and depends on the isotopy classes. Both intersection numbers have the same parity and satisfy the inequality

$$
|\langle a, b\rangle| \leq i(a, b) .
$$


We say that $a$ and $b$ are geometrically dual (respectively algebraically dual) if their geometric (respectively algebraic) intersection number is one.

A simple closed curve $a \subset \Sigma$ is called nonseparating if its complement is connected, otherwise it is called separating. Note that a simple closed curve is separating if and only if it is null-homologous (with either orientation) and thus simple closed curves that have geometric or algebraic duals are automatically nonseparating.

Diffeomorphisms of surfaces. Let us now turn to diffeomorphisms of surfaces. Let $\operatorname{Diff}^{+}(\Sigma, \partial \Sigma)$ denote the set of orientation-preserving diffeomorphisms that restrict to the identity on $\partial \Sigma$ and preserve the set of marked points. The mapping class group of $\Sigma$ is defined as

$$
\operatorname{Mod}(\Sigma):=\pi_{0}\left(\operatorname{Diff}^{+}(\Sigma, \partial \Sigma), \text { id }\right) .
$$

Given a simple closed curve $a \subset \Sigma$ there is a well defined mapping class $\tau_{a} \in \operatorname{Mod}(\Sigma)$ called the (right-handed) Dehn twist about $a$. Similarly, any simple $\operatorname{arc} r \subset \Sigma$ that connects two distinct marked points gives rise to a half twist $\bar{\tau}_{r} \in \operatorname{Mod}(\Sigma)$.

It is well known that $\operatorname{Mod}(\Sigma)$ is generated by the collection of Dehn twist and half twists, where the latter are only needed in the presence of marked points. On the other hand, mapping classes can be effectively studied by their action on (isotopy classes of) simple closed curves. In particular, it is desirable to understand the effect of Dehn twists on simple closed curves. While this can be tricky, the situation simplifies significantly on the level of homology classes.

Proposition 2.7 (Picard-Lefschetz formula). Let $\Sigma$ be a surface, $a \subset \Sigma$ a simple closed curve and let $x \in H_{1}(\Sigma)$. Then for any orientation on a we have

$$
\left(\tau_{a}^{k}\right)_{*} x=x+k\langle[a], x\rangle[a] .
$$

In particular, if $b$ is an oriented simple closed curve, then

$$
\left[\tau_{a}^{k}(b)\right]=[b]+k\langle[a],[b]\rangle[a] .
$$

Proof. See [Farb and Margalit 2011, Proposition 6.3]

Remark 2.8. The Picard-Lefschetz formula is particularly useful for the torus since, in that case, mapping classes are completely determined by their action on homology.

Another useful tool is the change of coordinates principle, which roughly states that any two configurations of simple closed curves on a surface with the same intersection pattern can be mapped onto each other by a diffeomorphism. We will only use the following special cases. For details we refer to [Farb and Margalit 2011, Chapter 1.3]. 
Proposition 2.9 (change of coordinates principle). If $a, b \subset \Sigma$ is a pair of nonseparating simple closed curves, then there exists some $\phi \in \operatorname{Diff}^{+}(\Sigma, \partial \Sigma)$ such that $\phi(a)=b$. Furthermore, if $a, b$ and $a^{\prime}, b^{\prime}$ are two pairs of geometrically dual curves, then there is some $\phi \in \operatorname{Diff}^{+}(\Sigma, \partial \Sigma)$ such that $\phi(a)=a^{\prime}$ and $\phi(b)=b^{\prime}$.

Mapping tori and their automorphisms. Given a surface $\Sigma$ and a diffeomorphism $\mu: \Sigma \rightarrow \Sigma$, possibly not orientable or orientation-preserving, we can form the mapping torus

$$
\Sigma(\mu):=(\Sigma \times[0,1]) /((x, 1) \sim(\mu(x), 0))
$$

which is a 3 -manifold that fibers over $S^{1} \cong[0,1] /\{0,1\}$ in the obvious way. If $\Sigma$ is oriented and $\mu$ is orientation-preserving, then our conventions stated in the introduction induce an orientation on $\Sigma(\mu)$. All surface bundles over $S^{1}$ can be described as mapping tori. Indeed, if a 3-manifold fibers over $S^{1}$, then one chooses a fiber and a lift of a vector field that determines the orientation of $S^{1}$ and the return map of the flow of this vector field induces a diffeomorphism of the fiber which is usually called the monodromy.

Let $Y$ be an oriented 3-manifold that fibers over the circle via a map $f: Y \rightarrow S^{1}$. An automorphism of $(Y, f)$ is an orientation- and fiber-preserving diffeomorphism of $Y$. We denote the group of automorphisms by $\operatorname{Aut}(Y, f)$ or simply by $\operatorname{Aut}(Y)$ when the fibration is clear from the context. If we identify $Y$ with a mapping torus, say $\Sigma(\mu)$, then we obtain a description of $\operatorname{Aut}(Y)$ in terms of diffeomorphisms of $\Sigma$. Indeed, any element $\phi \in \operatorname{Aut}(\Sigma(\mu))$ can be considered as a path $\left(\phi_{t}\right)_{t \in[0,1]}$ in $\operatorname{Diff}^{+}(\Sigma)$ connecting some element $\phi_{0} \in \operatorname{Diff}^{+}(\Sigma)$ to $\phi_{1}=\mu^{-1} \phi_{0} \mu$. In particular, $\phi_{0}$ must be isotopic to $\mu^{-1} \phi_{0} \mu$ and thus represents an element of $C_{\operatorname{Mod}(\Sigma)}(\mu)$, the centralizer in $\operatorname{Mod}(\Sigma)$ of (the mapping class represented by) $\mu$. Elaborating on this observation one arrives at the conclusion that

$$
\pi_{0}(\operatorname{Aut}(Y)) \cong \pi_{0}(\operatorname{Aut}(\Sigma(\mu))) \cong C_{\operatorname{Mod}(\Sigma)}(\mu) \ltimes \pi_{1}(\operatorname{Diff}(\Sigma), \mathrm{id}),
$$

where the multiplication on the right side is given by

$$
(g, \sigma) \cdot(h, \tau)=\left(h \circ g,\left(g^{-1} \tau g\right) * \sigma\right) .
$$

This means that there are essentially two types of automorphism of mapping tori: the ones that are constant on the fibers coming from $C_{\operatorname{Mod}(\Sigma)}(\mu)$ and the ones coming from $\pi_{1}(\operatorname{Diff}(\Sigma)$, id) that vary with the fibers and restrict to the identity on the reference fiber. However, it turns out that for most surfaces there are no nonconstant automorphisms.

Theorem 2.10 [Earle and Eells 1969]. If $\Sigma$ is a closed, orientable surface without marked points, the group $\pi_{1}(\operatorname{Diff}(\Sigma)$, id $)$ is isomorphic to $\mathbb{Z}_{2}, \mathbb{Z} \oplus \mathbb{Z}$, or the trivial group, depending on whether the genus $g$ equals 0,1 , or more than 1 . 
Hence, as soon as the genus of the fiber of a mapping torus is at least two, all automorphisms are isotopic (through automorphisms) to constant ones.

Remark 2.11. It is important not to confuse the group $\operatorname{Aut}(Y)$ with the group of all (orientation-preserving) diffeomorphisms of $Y$. A general diffeomorphism will not even be isotopic to a fiber-preserving one!

Theorem 2.10 has many important consequences, of which we only highlight one.

Corollary 2.12. Let $P \rightarrow S^{2}$ be a surface bundle with closed fibers of genus $g$.

(1) If $g=0$, then $P$ is diffeomorphic to $S^{2} \times S^{2}$ or $\mathbb{C} P^{2} \# \overline{\mathbb{C} P^{2}}$.

(2) If $g=1$, then $P$ is diffeomorphic to $T^{2} \times S^{2}, S^{1} \times S^{3}$ or $S^{1} \times L(n, 1)$.

(3) If $g \geq 2$, then $P$ is diffeomorphic to $\Sigma_{g} \times S^{2}$

Proof. For the genus-one case see [Baykur and Kamada 2010, Lemma 10]. The other cases are well known.

\section{Simple wrinkled fibrations over general base surfaces}

Without further ado we introduce the main objects of study in this paper.

Definition 3.1. Let $X$ be a 4-manifold and $B$ a surface, both oriented. A simple wrinkled fibration with total space $X$ and base $B$ is wrinkled fibration $w: X \rightarrow B$ with the following additional properties:

(1) $\partial X=w^{-1}(B)$.

(2) $\mathcal{C}_{w} \cap \partial X=\varnothing$.

(3) $\mathcal{C}_{w}$ is nonempty, connected, and contains a cusp.

(4) $w$ is injective on $\mathcal{C}_{w}$.

(5) All fibers of $w$ are connected.

The genus of $w$ is the maximal genus among all regular fibers. Finally, two simple wrinkled fibrations $w: X \rightarrow B$ and $w^{\prime}: X^{\prime} \rightarrow B^{\prime}$ are equivalent if there are orientation-preserving diffeomorphisms $\hat{\phi}: X \rightarrow X^{\prime}$ and $\breve{\phi}: B \rightarrow B^{\prime}$ such that $w^{\prime} \circ \hat{\phi}=\check{\phi} \circ w$.

A neighborhood of the critical image of a simple wrinkled fibration is shown in Figure 5. Before we continue we make some remarks about the definition.

Remark 3.2. Simple wrinkled fibrations over $S^{2}$ are, in essence, the same as Williams' simplified purely wrinkled fibrations, with two minor differences. On the one hand we do not put restrictions on the fiber genus, but on the other we require the presence of cusps. Both conditions can always be achieved by applying 


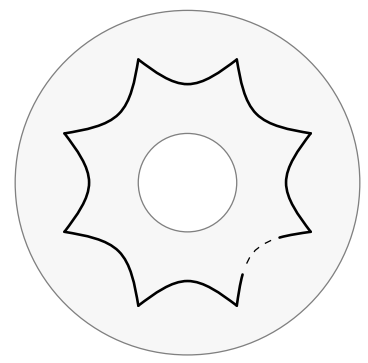

Figure 5. A neighborhood of the critical image of a simple wrinkled fibration.
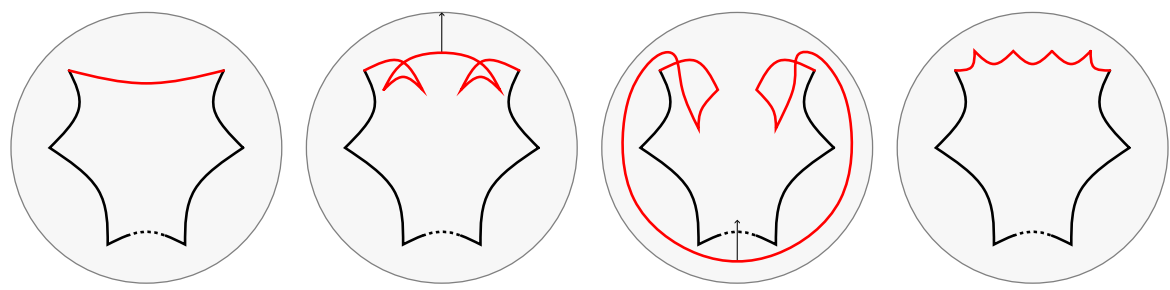

Figure 6. The base diagrams during a flip-and-slip move. (The pictures show the complement of a disk in the lower-genus region of the original fibration.)

a flip-and-slip move (see next remark) and are thus not restrictive. Moreover, the "simple wrinkled fibrations without cusps" are easily classified (see Example 3.7), so one does not lose too much by excluding them.

Remark 3.3. Given a simple wrinkled fibration over $S^{2}$ there is an important homotopy to another such simple wrinkled fibration which has become known as a flip-and-slip move. Its effect on the base diagram is shown in Figure 6. One first perform two flips on the same fold arc and then chooses an isotopy of the total space (the slip) during which the critical image undergoes the changes demonstrated in the picture. A flip-and-slip increases the fiber genus by one and introduces four new cusps.

Remark 3.4. In spite of the lengthy definition, simple wrinkled fibrations are arguably the simplest possible maps from 4-manifolds to surfaces, at least as far as their singularity structure is concerned. As will be explained in detail it is this simplicity which makes it possible to give nice combinatorial descriptions of 4-manifolds.

Given the rather specialized nature of simple wrinkled fibrations one might wonder whether they actually exist. This is indeed the case and we begin by giving some simple constructions. 
Example 3.5 (surface bundles). Let $\pi: X \rightarrow B$ be a surface bundle over a surface $B$ with closed fibers of genus $g$. Then we can perform a birth homotopy on $\pi$ to obtain a simple wrinkled fibration of genus $g+1$ with two cusps.

Example 3.6 (Lefschetz fibrations). If $f: X \rightarrow B$ is a Lefschetz fibration (possibly achiral) with closed fibers of genus $g$, then after wrinkling all the Lefschetz singularities we obtain a number of disjoint circles with three cusps in the critical image. By suitably merging cusps we can turn this configuration into a single circle resulting in a simple wrinkled fibration of genus $g+1$.

Example 3.7 (the case without cusps). This example includes the broken Lefschetz fibration on $S^{4}$ from [Auroux et al. 2005] that was mentioned in the introduction. Let $\Omega$ be a cobordism from $\Sigma_{g}$ to $\Sigma_{g-1}$ together with a Morse function $\mu: \Omega \rightarrow I$ with exactly one critical point of index two. Then $\mu \times \mathrm{id}: \Omega \times S^{1} \rightarrow I \times S^{1}$ is a stable map with one circle of indefinite folds which fails to be a simple wrinkled fibration only because it does not have any cusps. Nevertheless, we can use $\Omega \times S^{1}$ to build wrinkled fibrations over $S^{2}$ by suitably filling in the two boundary components with $\Sigma_{g} \times D^{2}$ and $\Sigma_{g-1} \times D^{2}$ such that the fibration structures on the boundary extends. Using the handle decomposition from [Baykur 2009] it is easy to see that this construction produces the following total spaces: $P \# S^{1} \times S^{3}$ where $P$ is any $\Sigma_{g-1}$-bundle over $S^{2}$ and, if $g=1, S^{4}$ and some other manifolds with finite cyclic fundamental group; see [Baykur and Kamada 2010; Hayano 2011]. Having built these maps one can then apply a flip-and-slip to obtain honest simple wrinkled fibrations. In particular, $S^{4}$ carries a simple wrinkled fibration of genus two.

These examples show that simple wrinkled fibrations can be considered as a common generalization of surface bundles and (achiral) Lefschetz fibrations. The vastness of this generalization is indicated by the following remarkable theorem.

Theorem 3.8 [Williams 2010]. Let $X$ be a closed, oriented 4-manifold. Then any map $X \rightarrow S^{2}$ is homotopic to a simple wrinkled fibration of arbitrarily high genus.

Remark 3.9. Williams' proof builds on the results of [Gay and Kirby 2007] which, in turn, depends on deep theorems in 3-dimensional contact topology. This somewhat unnatural dependence could be removed by refining the singularity theory based approach of [Baykur 2008] to produce maps which are injective on their critical locus.

Williams [2010] introduced a combinatorial description of simple wrinkled fibrations over $S^{2}$ in terms of what he calls surface diagrams. We will generalize his construction to the setting of general base surfaces.

Let $w: X \rightarrow B$ be a simple wrinkled fibration. The discussion in Section 2A shows that the critical locus $\mathcal{C}_{w} \subset X$ is a smoothly embedded circle and that $w$ restricts to a topological embedding of $\mathcal{C}_{w}$ into $B$. Furthermore, the critical 
image $w\left(\mathcal{C}_{w}\right)$ separates $B$ into two components. Indeed, if the complement were connected, then all regular fibers would be diffeomorphic. But according to the fold model, the topology of the fibers on the two sides of a fold arc must be different. In fact, since we require that all fibers are connected, the genus on one side has to be one higher than on the other side. We will call the two components of $B \backslash w\left(\mathcal{C}_{w}\right)$ the higher- and lower-genus regions.

We would like to understand more precisely how the topology of the fibers changes across the critical image. A reference path for $w$ is an oriented, embedded arc $R \subset B$ that connects a point $p_{+}$in the higher-genus region to a point $p_{-}$in the lower-genus region and intersects $w\left(\mathcal{C}_{w}\right)$ transversely in exactly one fold point. Then the reference fibers $\Sigma_{ \pm}(R):=w^{-1}\left(p_{ \pm}\right)$over the reference points $p_{ \pm}$are closed, oriented surfaces.

Lemma 3.10. A reference path $R \subset B$ induces a nonseparating simple closed curve $c(R) \subset \Sigma_{+}(R)$ which depends only on the isotopy class of $R$ relative to its reference points and the cusps.

Definition 3.11. The curve $c(R) \subset \Sigma_{+}(R)$ is called the (fold) vanishing cycle associated to $R$.

Proof. The fold model implies that $w^{-1}(R)$ is a cobordism from $\Sigma_{+}(R)$ to $\Sigma_{-}(R)$ on which $w$ restricts to a Morse function with exactly one critical point of index 2 . Thus $w^{-1}(R)$ is diffeomorphic to $\Sigma_{+}(R) \times[0,1]$ with a (3-dimensional) 2-handle attached along a simple closed curve in $\Sigma_{+}(R) \times\{1\}$ which is canonically identified with a simple closed curve $c(R) \subset \Sigma_{+}(R)$.

Next, let us look at what happens around the cusp. Let $R_{1}$ and $R_{2}$ be two reference paths for $w$ with common reference points and assume that their interiors are disjoint. We call $R_{1}$ and $R_{2}$ adjacent if their union $R_{1} \cup R_{2}$ bounds a disk in $B$ that contains exactly one cusp.

Lemma 3.12. Let $R_{1}$ and $R_{2}$ be adjacent reference paths. Then the vanishing cycles $c\left(R_{1}\right)$ and $c\left(R_{2}\right)$ in $\Sigma_{+}:=\Sigma_{+}\left(R_{1}\right)=\Sigma_{+}\left(R_{2}\right)$ are geometrically dual.

Proof. As in the proof of Lemma 3.10 the preimages $w^{-1}\left(R_{i}\right), i=1,2$, are both cobordisms from $\Sigma_{+}$to $\Sigma_{-}$, each consisting of a 2-handle attachment along $c\left(R_{i}\right)$. By reversing the orientation of $R_{1}$ we can consider $w^{-1}\left(R_{1}\right)$ as a cobordism from $\Sigma_{-}$to $\Sigma_{+}$, now consisting of a 1-handle attachment. In this process the former attaching sphere of the 2-handle $c\left(R_{1}\right)$ becomes the belt sphere of the 1-handle.

Gluing $w^{-1}\left(R_{1}\right)$ and $w^{-1}\left(R_{2}\right)$ together along $\Sigma_{+}$gives a cobordism from $\Sigma_{-}$to itself consisting of a 1-handle attachment followed by a 2-handle attachment. Now recall that a cusp models the death (or birth) of a canceling pair of Morse critical points. Hence, the attaching sphere of the 2-handle, which is $c\left(R_{2}\right)$, intersects the belt sphere of the 1-handle, which is $c\left(R_{1}\right)$, in a single point. 
Looking a bit ahead, our strategy will be to choose suitable collections of reference paths and to study simple wrinkled fibrations in terms of the induced collection of vanishing cycles. The only obstacle for doing so is the possibly complicated topology of the base surface. But this can easily be overcome by cutting the base into three pieces

$$
B=B_{+} \cup A \cup B_{-}
$$

where $A$ is a regular neighborhood of the critical image of $w$ (diffeomorphic to an annulus) and $B_{ \pm}$are the closures of the complement of $A$. The subscript in $B_{ \pm}$ indicates whether the surface is contained in the higher- or lower-genus region. Note that $w$ restricts to surface bundles over $B_{ \pm}$and, although complicated, these form a rather well studied class of objects. Thus the interesting new part of $w$ is the restriction $w^{-1}(A) \rightarrow A$ which is a simple wrinkled fibration over an annulus whose critical image is boundary parallel.

Definition 3.13. A simple wrinkled fibration $w: W \rightarrow A$ over an annulus $A$ is called annular if its critical image is boundary parallel.

So in order to understand simple wrinkled fibrations over any base surface, it is enough to understand annular simple wrinkled fibrations and this is where twisted surface diagrams (see Definition 3.20 below) enter the picture. The remainder of this section is devoted to the proof of Theorem 1.1 stated in the introduction.

Remark 3.14. Gay and Kirby [2012] have published a result that contains Theorem 1.1 as a special case. Although their methods are somewhat similar to ours we feel that our approach is of independent interest.

We will split the proof of the theorem into the two obvious parts. The first part is the subject of Section 3A (see Proposition 3.25) where we show how assign twisted surface diagrams to annular simple wrinkled fibrations. The second part that shows how to build annular simple wrinkled fibrations from twisted surface diagrams is treated in Section 3C (see Proposition 3.31). In between, we will see in Section 3B that, just as Lefschetz fibrations, annular simple wrinkled fibrations are directly accessible via handlebody theory.

\section{A. Twisted surface diagrams of annular simple wrinkled fibrations. Consider} an annular simple wrinkled fibration $w: W \rightarrow A$. We denote by $\partial_{+} A$ and $\partial_{-} A$ the boundary components of the base annulus $A$ contained in the higher- and lower-genus regions, respectively, and we let

$$
\partial_{ \pm} W=w^{-1}\left(\partial_{ \pm} A\right) .
$$

We equip $\partial_{+} A$ and $\partial_{+} W$ with the opposite boundary orientation, so that $W$ is an oriented cobordism from $\partial_{+} W$ to $\partial_{-} W$. 

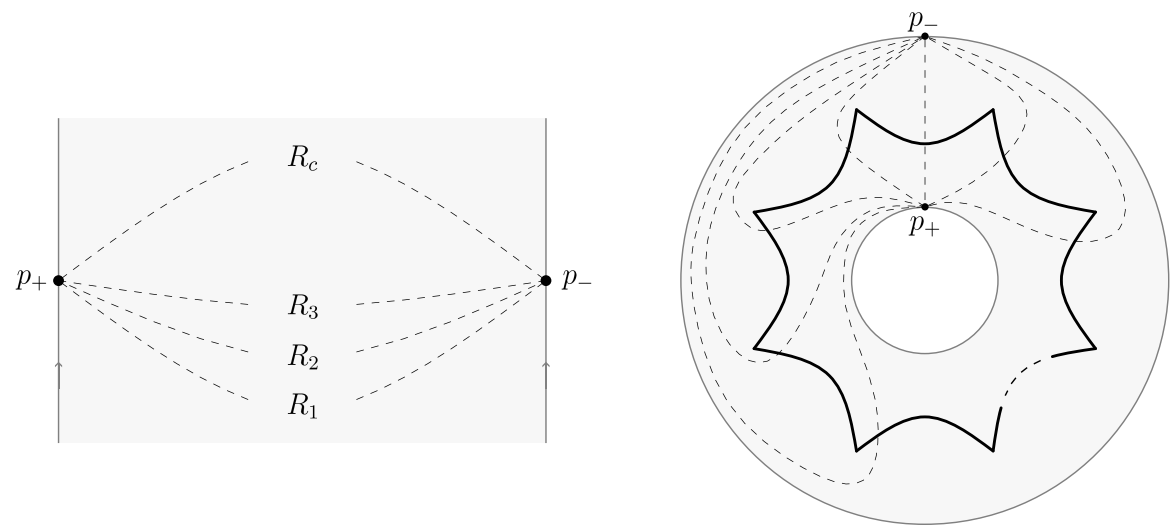

Figure 7. A reference system for an annular simple wrinkled fibration.

Definition 3.15. Let $w: W \rightarrow A$ be an annular simple wrinkled fibration. A reference system for $w$ is a collection of reference paths $\mathcal{R}=\left\{R_{1}, \ldots, R_{l}\right\}$ for $w$ (where $l$ is the number of cusps) such that

(1) all reference paths have the same reference points $p_{ \pm} \in \partial_{ \pm} A$,

(2) the interiors of the arcs are pairwise disjoint,

(3) with respect to the orientations on $\partial_{ \pm} A$ the arcs leave $\partial_{+} A$ and enter $\partial_{-} A$ in order of increasing index (see Figure 7) and

(4) each fold arc is hit by exactly one of the $R_{i}$.

Remark 3.16. Condition (3) might need some further explanation. Assume that we have a collection of properly embedded arcs in a surface which all hit the boundary in the same point and are otherwise disjoint near that boundary component. If the boundary component is oriented, then there is a well defined notion of order for the arcs which can be described as follows. We take a small half disk around the boundary point and orient the boundary of this half disk so that it agrees with the orientation of the boundary component of the surface. For a generic choice of half disk each arc will intersect the boundary of the half disk transversely in one point and the order of these intersection points is easily seen to be independent of the choice of half disk.

As before, we denote the reference fibers by $\Sigma_{ \pm}:=\Sigma_{ \pm}(\mathcal{R})=w^{-1}\left(p_{ \pm}\right)$. Using the reference fibers we can write $\partial_{ \pm} W$ as mapping tori

$$
\partial_{ \pm} W \cong \Sigma_{ \pm}\left(\mu_{ \pm}\right)
$$

where $\mu_{ \pm} \in \operatorname{Mod}\left(\Sigma_{ \pm}\right)$is the monodromy of $w$ over $\partial_{ \pm} A$ (in the positive direction). We will refer to $\mu_{+}$and $\mu_{-}$as the higher and lower monodromies of $w$. 


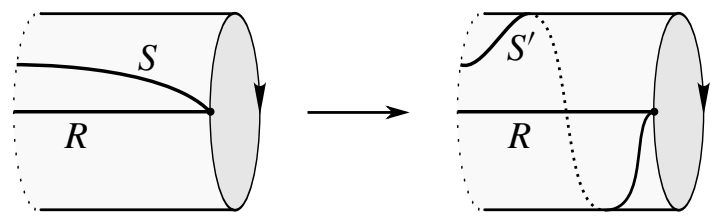

Figure 8. Swinging an arc around a boundary component.

Lemma 3.17. Let $w: W \rightarrow A$ be an annular simple wrinkled fibration together with a reference system $\mathcal{R}=\left\{R_{1}, \ldots, R_{l}\right\}$ and let $c_{i}=c\left(R_{i}\right) \subset \Sigma_{+}$. For $i<l$ the vanishing cycles $c_{i}$ and $c_{i+1}$ are geometrically dual, and so are $\mu_{+}\left(c_{l}\right)$ and $c_{1}$.

In the proof we need the following construction. Let $B$ be an oriented surface and let $R$ and $S$ be two properly embedded arcs in $B$ which hit a boundary component $\partial_{i} B \subset \partial B$ transversely in the same point such that $S$ enters $\partial_{i} B$ after $R$ (as explained in Remark 3.16) and whose interiors are disjoint. As indicated in Figure 8 we can modify $S$ by moving its endpoint along $\partial_{i} B$ resulting in a new $\operatorname{arc} S^{\prime}$ which enters $\partial_{i} B$ before $R$ and whose interior is still disjoint from $R$. We will say that $S^{\prime}$ is obtained from $S$ be swinging once around $\partial_{i} B$. (Note that swinging around $\partial_{i} B$ is not the same as performing a boundary parallel Dehn twist since such Dehn twists are supported in the interior of $B$ and fix a collar neighborhood of the boundary. In particular, they cannot change the order of arcs at the boundary and, moreover, in Figure 8 a boundary parallel Dehn twist applied to $S$ would produce an arc that intersects $R$ in its interior.)

Proof of Lemma 3.17. The first statement follows from Lemma 3.12 since for $i<l$ the reference paths $R_{i}$ and $R_{i+1}$ are clearly adjacent. For the second statement we first swing $R_{l}$ once around the boundary of $A$ so that the resulting reference path $R_{l}^{\prime}$ is adjacent to $R_{1}$ and thus $c\left(R_{l}^{\prime}\right)$ is geometrically dual to $c\left(R_{1}\right)$. Next we observe that $R_{l}^{\prime}$ is homotopic to $R_{l}$ precomposed with the boundary curve. Thus the parallel transport along $R_{l}^{\prime}$ is the composition of the parallel transport along $R_{l}$ and the higher-genus monodromy. In particular, we have $c\left(R_{l}^{\prime}\right)=\mu_{+}\left(c_{l}\right)$.

Remark 3.18. Note that in the above proof we did not actually need the whole reference system but only the parts of the arcs contained in the higher-genus region.

Let us isolate the combinatorial structure encountered in the above lemma.

Definition 3.19. Let $\Sigma$ be a surface. A circuit (of length $l$ ) on $\Sigma$ is an ordered collection of simple closed curves $\Gamma=\left(c_{1}, \ldots, c_{l}\right)$ such that any two adjacent curves $c_{i}$ and $c_{i+1}$ are geometrically dual for $i<l$. A switch for $\Gamma$ is a mapping class $\mu \in \operatorname{Mod}(\Sigma)$ such that $\mu\left(c_{l}\right)$ and $c_{1}$ are geometrically dual. We say that $\Gamma$ is closed if $c_{l}$ and $c_{1}$ are geometrically dual, that is, if the identity works as a switch. 
Definition 3.20. A twisted surface diagram is a triple $\mathfrak{S}=(\Sigma, \Gamma, \mu)$ where $\Sigma$ is a closed, oriented surface, $\Gamma$ is a circuit in $\Sigma$ and $\mu \in \operatorname{Mod}(\Sigma)$ is a switch for $\Gamma$. In the case that $\Gamma$ is a closed circuit and $\mu=\mathrm{id}$, we simply speak of surface diagrams and shorten the notation to $\mathfrak{S}=(\Sigma, \Gamma)$ or sometimes even $\left(\Sigma ; c_{1}, \ldots, c_{l}\right)$.

Remark 3.21. Note that our definition of surface diagrams is slightly different from Williams' original definition [2010]. Indeed, Williams requires that surface diagrams are induced from simple wrinkled fibrations over the sphere so that the associated annular simple wrinkled fibration has trivial higher and lower monodromies while we only require trivial higher monodromy. The reason for our deviance is that we would like to have an abstract definition of (twisted) surface diagram that does not depend on any relation to simple wrinkled fibrations. However, it turns out that the trivial lower monodromy condition for an annular simple wrinkled fibration is not easy to state in terms of its twisted surface diagrams (see Section 4A for the untwisted case) and we find it more appropriate to consider it as an extra condition.

Remark 3.22. There is no restriction on the intersections of nonadjacent curves in a circuit. Circuits in which nonadjacent curves are disjoint, so called chains of curves, are well known objects in the theory of mapping class groups of surfaces where they play an important role.

Remark 3.23. Sometimes it will be convenient to choose orientations on the curves in a circuit $\Gamma=\left(c_{1}, \ldots, c_{l}\right)$ in order to speak of their homology classes. If the ambient surface is oriented, we always choose orientations such that the intersection of $c_{i}$ and $c_{i+1}, i<l$, has positive sign.

With this terminology we can rephrase Lemma 3.17 as stating that an annular simple wrinkled fibration $w: W \rightarrow A$ together with a reference system $\mathcal{R}$ induces a twisted surface diagram

$$
\mathfrak{S}_{w, \mathcal{R}}:=\left(\Sigma_{+}, \Gamma_{w, \mathcal{R}}, \mu_{+}\right)
$$

where the higher monodromy works as a switch.

Not surprisingly, the twisted surface diagrams constructed in Lemma 3.17 depend on the choice of the reference system. To understand this dependence we observe that a reference system is uniquely determined (up to isotopy relative to the boundary and the cusps) by specifying the first reference path - this follows directly from the definition. Furthermore, it is easy to see that any two reference paths which have the same reference points and hit the same fold arc become isotopic after suitably swinging around the boundary components of $A$.

Now let $\mathcal{R}=\left\{R_{1}, \ldots, R_{l}\right\}$ and $\mathcal{S}=\left\{S_{1}, \ldots, S_{l}\right\}$ be two reference systems with common reference points and let $S_{k}$ hit the same fold arc as $R_{1}$. As in the proof of Lemma 3.17 we successively swing the arcs $S_{l}, S_{l-1}, \ldots, S_{k}$ once around each boundary component to obtain a new reference system $\mathcal{S}^{\prime}$ in which the first reference 
path hits the same fold arc as $R_{1}$. Now, by further swinging all of $\mathcal{S}^{\prime}$ simultaneously, but this time independently around the boundary components, we can match the two first reference paths and thus the whole reference systems.

Let us analyze the effect of this matching procedure on the twisted surface diagram. For brevity of notation let $\mathfrak{S}=(\Sigma, \Gamma, \mu)$ be the twisted surface diagram associated to an annular simple wrinkled fibration $w: W \rightarrow A$ together with a reference system $\mathcal{R}$. Since the surface $\Sigma$ and the switch $\mu$ only depend on the reference points, only the circuit $\Gamma=\left(c_{1}, \ldots, c_{l}\right)$ will be affected by swinging some reference paths. Moreover, note again that the vanishing cycles $c_{i}$ only depend on the part of the reference paths contained in the higher-genus region. Thus swinging around the lower-genus boundary does not change the circuit.

Now, as we have already observed, if we swing the last reference path in $\mathcal{R}$ once around both boundary components, we obtain a new reference system $\mathcal{R}^{\prime}$, which induces the circuit

$$
\Gamma_{\mu}^{[1]}:=\left(\mu\left(c_{l}\right), c_{1}, \ldots, c_{l-1}\right) .
$$

This operation of going from $\mathfrak{S}$ to $\mathfrak{S}^{[1]}:=\left(\Sigma, \Gamma_{\mu}^{[1]}, \mu\right)$ makes sense in the abstract setting of twisted surface diagrams and we call it (and its obvious inverse) switching. Note that if the higher monodromy $\mu$ is trivial, then switching simply amounts to a cyclic permutation of the vanishing cycles.

Since we can relate any two reference systems for a given annular simple wrinkled fibration by suitably swinging reference paths, we see that the twisted surface diagram is well defined up to switching.

Next we want to compare the twisted surface diagrams of two equivalent annular simple wrinkled fibrations as in the commutative diagram below.

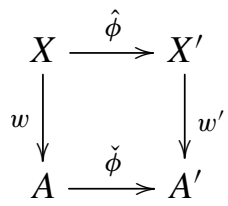

If $\mathcal{R}$ is a reference system for $w$, then $\mathcal{R}^{\prime}:=\check{\phi}(\mathcal{R})$ is a reference system for $w^{\prime}$. Let $\mathfrak{S}=(\Sigma, \Gamma, \mu)$ and $\mathfrak{S}^{\prime}=\left(\Sigma^{\prime}, \Gamma^{\prime}, \mu^{\prime}\right)$ be the associated twisted surface diagrams. Then $\hat{\phi}$ induces an orientation-preserving diffeomorphism $\phi: \Sigma \rightarrow \Sigma^{\prime}$ and clearly the higher monodromies satisfy $\mu^{\prime}=\phi \mu \phi^{-1}$. It is also easy to see that

$$
\Gamma^{\prime}=\phi(\Gamma):=\left(\phi\left(c_{1}\right), \ldots, \phi\left(c_{l}\right)\right)
$$

where, as usual, $\Gamma=\left(c_{1}, \ldots, c_{l}\right)$. Again, the effect of an equivalence of annular simple wrinkled fibrations makes sense for abstract twisted surface diagrams and we say that $\mathfrak{S}$ and $\mathfrak{S}^{\prime}$ are diffeomorphic via $\phi$. 
Combining this with switching we end up with the following definition.

Definition 3.24. Two twisted surface diagrams $\mathfrak{S}$ and $\mathfrak{S}^{\prime}$ called equivalent if, for some integer $k, \mathfrak{S}^{\prime}$ is diffeomorphic to $\mathfrak{S}^{[k]}$.

Summarizing this section so far, we have proved the first half of Theorem 1.1:

Proposition 3.25. To an annular simple wrinkled fibration $w: W \rightarrow A$ we can assign a twisted surface diagram

$$
\mathfrak{S}_{w}=\left(\Sigma_{+}, \Gamma_{w}, \mu_{+}\right)
$$

which is well defined up to equivalence. Moreover, equivalent annular simple wrinkled fibrations have equivalent twisted surface diagram.

Remark 3.26. We would like to point out that it is very convenient that only the equivalence class of the surface diagram plays a role. Indeed, in order to actually visualize the twisted surface diagram of an annular simple wrinkled fibration one has to identify the higher-genus reference fiber with some model surface and there is no canonical way to do so. However, any two such identifications will differ by a diffeomorphism of the model surface and thus be equivalent. So we can safely forget about the choice of identification whenever we are only interested in the equivalence class of the simple wrinkled fibrations or the diffeomorphism type of its total space.

3B. Handle decompositions for annular simple wrinkled fibrations. As a next step we relate the twisted surface diagrams associated to annular simple wrinkled fibrations to the topology of their total spaces. We will see that the situation is very similar to Lefschetz fibrations.

Proposition 3.27. Let $w: W \rightarrow A$ be an annular simple wrinkled fibration. Then $W$ has a relative handle decomposition on $\partial_{+} W$ with one 2-handle for each fold arc. Such a handle decomposition is encoded in any twisted surface diagram for $w$.

In the following we will refer to the 2-handles associated to the fold arcs as fold handles.

Proof. The rough idea is to parametrize $A$ by $S^{1} \times[0,1]$ such that the composition of $w$ and the projection $p: S^{1} \times[0,1] \rightarrow[0,1]$ becomes a Morse function. We equip $S^{1} \times[0,1]$ with coordinates $(\theta, t)$ and refer to the direction in which $t$ increases as right. We say that a parametrization $\kappa: A \rightarrow S^{1} \times[0,1]$ is $w$-regular if the critical image $C_{\kappa}:=\kappa \circ w\left(\mathcal{C}_{w}\right)$ is in the following standard position:

- All cusps point to the right.

- Each $R_{\theta}:=\{\theta\} \times[0,1]$ meets $C_{\kappa}$ in exactly one point, either in a cusp or transversely in a fold point.

- The projection $p$ restricted to $C_{\kappa}$ has exactly one minimum on each fold arc. 
We claim that for any $w$-regular parametrization $\kappa$, the map

$$
p_{\kappa}:=p \circ \kappa \circ w: W \rightarrow[0,1]
$$

is a Morse function. Clearly, the critical points of $p_{\kappa}$ are contained in $\mathcal{C}_{w}$. Thus we have to understand how the projection $p$ interacts with the critical image $C_{\kappa}$. By the standard position assumption there are three ways how a level set $S_{t}:=S^{1} \times\{t\}$ can intersect $C_{\kappa}$ (see Figure 9):

a) $S_{t}$ intersects $C_{\kappa}$ transversely in a fold point,

b) $S_{t}$ meets $C_{\kappa}$ in a cusp and the fold arcs surrounding the cusp are on the left side of $S_{t}$ or

c) $S_{t}$ is tangent to a fold arc which is located on the right side of $S_{t}$. We will refer to this phenomenon as a left tangency.

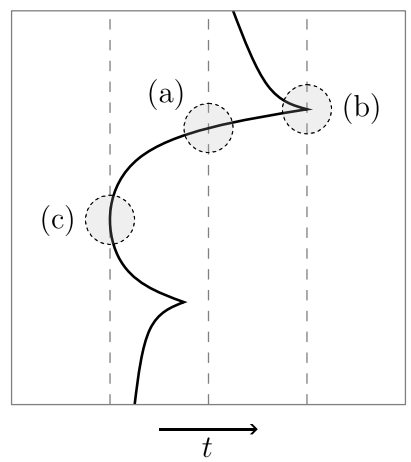

Figure 9. Level sets intersecting the critical image.

It turns out that only the left tangencies contribute critical points of $p_{\kappa}$. In fact, from the models for the fold and cusp we immediately see that $p_{\kappa}$ is modeled on the compositions

$$
(t, x, y, z) \mapsto\left(t,-x^{3}+3 t x-y^{2}+z^{2}\right) \mapsto t
$$

in case of a cusp intersection and

$$
(t, x, y, z) \mapsto\left(t,-x^{2}-y^{2}+z^{2}\right) \mapsto \pm t
$$

for a transverse fold intersection (the sign depends on how the fold and cusp models are embedded) which shows that these are regular points of $p_{\kappa}$.

It remains to treat the concave tangencies. These occur precisely at the minima of $\left.p_{\kappa}\right|_{C_{\kappa}}$. This minimum can be modeled by $t \mapsto t^{2}$ and it is easy to see that $p_{\kappa}$ is modeled on

$$
(t, x, y, z) \mapsto\left(-x^{2}-y^{2}+z^{2}+t^{2}\right)
$$


which is a Morse critical point of index 2. By assumption there is exactly one concave tangency for each fold arc and, using the correspondence between Morse functions and handle decompositions, we obtain the desired handle decomposition.

In order to understand how the fold handles are attached, consider the arcs

$$
R_{i}:=R_{\theta_{i}} \subset S^{1} \times[0,1],
$$

where $\theta_{1}, \ldots, \theta_{l} \in S^{1}$ is a sequence of numbers ordered according to the orientation of $S^{1}$ (for example, the $l$-th roots of unity). The $w$-regular parametrization $\kappa$ can be chosen in such a way that each $R_{i}$ is a reference path for precisely one fold arc and $C_{\kappa}$ is contained in the open annulus $S^{1} \times(\epsilon, 1-\epsilon)$ for some $\epsilon>0$. For each $R_{i}$ we obtain a vanishing cycle $c_{i}$ in the fiber of $w$ over $\left(\theta_{i}, 0\right) \in \partial_{+} A$ and the local model for folds implies that the fold handles are attached to $\partial_{+} W \times[0, \epsilon]$ along the vanishing cycles $c_{i}$ pushed off into the fiber over $\left(\theta_{i}, \epsilon\right)$ with respect to the canonical framing induced by the fiber.

The relation to twisted surface diagrams now becomes obvious. There is a canonical way to turn the reference paths $\Theta_{1}, \ldots, \Theta_{l}$ into a reference system by fixing $\Theta_{1}$ and successively sliding the endpoints of the remaining arcs along the boundary onto $\Theta_{1}$ against the orientation. Thus the vanishing cycles record the attaching curves of the fold handles.

Remark 3.28. The above proposition is one of the reasons why we require the presence of cusps in the critical loci of simple wrinkled fibrations. If there were no cusps, then it would not be possible to avoid right tangencies which would correspond to 3-handles instead of 2-handles. Thus the presence of cusps guarantees that the total spaces of annular simple wrinkled fibrations are (relative) 2-handlebodies.

Remark 3.29. The observation that fold tangencies correspond to Morse critical points was also made by Gay and Kirby [2011a] in their more general setting of Morse 2-functions. The fact that the real part of the Lefschetz model is also a Morse function allows to include Lefschetz singularities in the discussion. Proceeding this way, one can recover Baykur's result [2009] about handle decompositions from broken Lefschetz fibrations.

Remark 3.30. The reader familiar with Lefschetz fibrations will have noticed the strong resemblance of the handle decompositions described above with the ones induced by Lefschetz fibrations. In fact, the handle decompositions have exactly the same structure except that the fold handles are attached with respect to the fiber framing while the framing of the Lefschetz handles differs by -1 .

3C. Annular simple wrinkled fibrations from twisted surface diagrams. Using the handle decompositions exhibited in the previous section as a stepping stone we can now build annular simple wrinkled fibrations out of twisted surface diagrams and thus complete the proof of Theorem 1.1. 

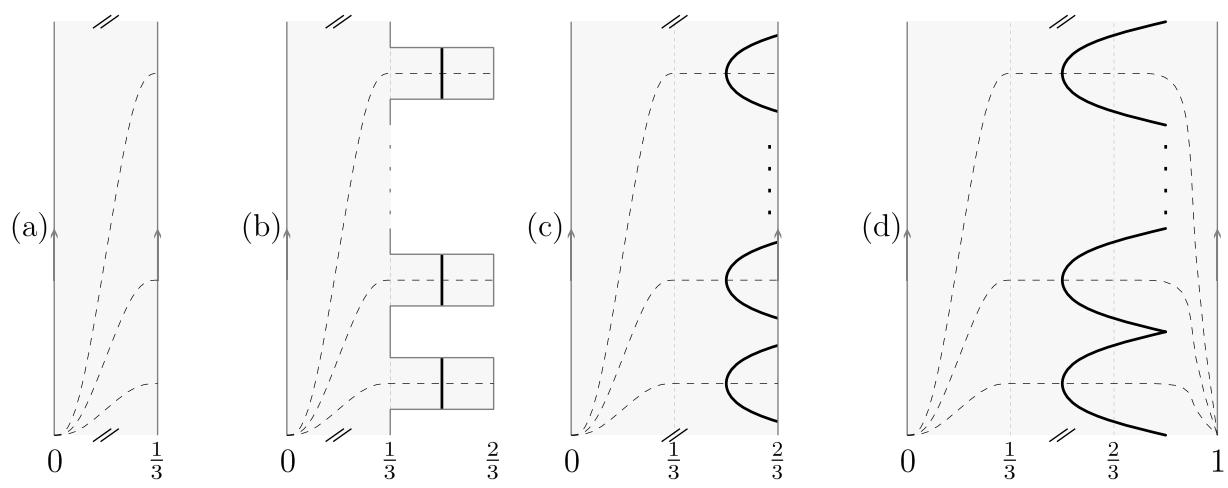

Figure 10. Building a simple wrinkled fibration from a surface diagram. Bold curves represent the critical image, and dashed curves the reference path.

Proposition 3.31. A twisted surface diagram $\mathfrak{S}=(\Sigma, \Gamma, \mu)$ determines an annular simple wrinkled fibration $w_{\mathfrak{S}}: W_{\mathfrak{S}} \rightarrow S^{1} \times[0,1]$ with higher-genus fiber $\Sigma$ and higher monodromy $\mu$.

Proof. To make the construction of $w_{\mathfrak{S}}$ more transparent we begin with some preliminary considerations. One important ingredient is the mapping cylinder $\Sigma(\mu)$ with its canonical fibration $p: \Sigma(\mu) \rightarrow S^{1}=[0,1] /\{0,1\}$ in which we identify $\Sigma$ with the fiber over $0 \sim 1$. We will also need a collection of $\operatorname{arcs} \mathcal{R}=\left\{R_{1}, \ldots, R_{l}\right\}$ in $S^{1} \times[0,1]$ that will serve as a reference system for $w_{\mathfrak{S}}$; see Figure 10(a). To construct these let $r:[0,1] \rightarrow[0,1]$ be a smooth function that has the constant value 1 on the interval $\left[\frac{1}{3}, \frac{2}{3}\right]$, satisfies $r(0)=r(1)=0$ and is strictly increasing for $t \leq \frac{1}{3}$ and strictly decreasing for $t \geq \frac{2}{3}$. If the length of $\Gamma$ is $l$, then for $i=1, \ldots, l$ we let $\theta_{i}:=(i-1) / c$ and define

$$
R_{i}:=\left\{\left(\theta_{i} r(t), t\right) / \sim \mid t \in[0,1] \subset S^{1} \times[0,1]\right\} .
$$

We can now give the construction of $W_{\mathfrak{S}}$ and $w_{\mathfrak{S}}$ in three steps.

Step 1: We begin by taking the product $W_{1}:=\Sigma(\mu) \times\left[0, \frac{1}{3}\right]$ and define a map $w_{1}: W_{1} \rightarrow S^{1} \times\left[0, \frac{1}{3}\right]$ by sending $(x, t)$ to $(p(x), t)$.

Step 2: Next, we construct $W_{2}$ by attaching 2-handles to $W_{1}$ in the following way. Let $\Gamma=\left(c_{1}, \ldots, c_{l}\right)$. Using the arc $R_{i} \subset S^{1} \times[0,1]$ described above we can parallel transport the curve $c_{i} \subset \Sigma$ to the fiber of $w_{1}$ over $\left(\theta_{i}, \frac{1}{3}\right)$. We attach a 2-handle to the resulting curve with respect to the fiber framing.

This choice of framing allows us to extend $w_{1}$ over each 2-handle. Indeed, we can consider attaching the $i$-th (4-dimensional) 2-handle as a 1-parameter family of 3-dimensional 2-handle attachments parametrized by a small neighborhood 
of $\left(\theta_{i}, 1\right)$ in $S^{1} \times\{1\}$. (Of course, these neighborhoods are pairwise disjoint.) For each point $\theta$ in such a neighborhood, the restriction of $w_{1}$ to the $\theta$-ray $\{\theta\} \times\left[0, \frac{1}{3}\right]$ extends to a Morse function (with one critical point of index 2) over a slightly longer ray, say $\{\theta\} \times\left[0, \frac{2}{3}\right]$, in the standard way. Using these 1-parameter families of Morse functions we can extend $w_{1}$ to map from $W_{2}$ to an annulus with "bumps" on one side, as shown in Figure 10(b), and this map has an arc of indefinite folds on each bump. We can then smooth out the bumps by standard techniques from differential topology to obtain a map $w_{2}: W_{2} \rightarrow S^{1} \times\left[0, \frac{2}{3}\right]$ in which each 2-handle attachment has created an arc of indefinite folds whose endpoints hit the boundary of $W_{2}$ transversely in the component that was affected by the handle attachment, as in Figure 10(c); let us call this component $\partial_{2} W_{2}$.

Step 3: For the final step we first note that the restriction of $w_{2}$ over $S^{1} \times\left\{\frac{2}{3}\right\}$ is a circle valued Morse function with a pair of critical points of index 1 and 2 for each fold arc of $w_{2}$. The crucial observation is that the condition that $\Gamma$ is a circuit with switch $\mu$ implies that all these pairs of critical points cancel! Thus there is a standard homotopy, which we parametrize by $\left[\frac{2}{3}, 1\right]$, from $\left.w_{2}\right|_{\partial_{2} W_{2}}$ to a submersion that realizes this cancellation. We let

$$
W_{\mathfrak{S}}:=W_{2} \cup_{\partial_{2} W_{2}} \partial_{2} W_{2} \times\left[\frac{2}{3}, 1\right]
$$

and extend $w_{2}$ over the newly added collar of $\partial_{2} W_{2}$ by tracing out the homotopy to obtain a map $w_{\mathfrak{S}}: W_{\mathfrak{S}} \rightarrow S^{1} \times[0,1]$. This last step removes all critical points from the boundary in exchange for an interior cusp for each canceling pair. Clearly $w_{\mathfrak{S}}$ is an annular simple wrinkled fibration with base diagram as in Figure 10(d).

Note that $W_{\mathfrak{S}}$ is diffeomorphic to $W_{2}$ and thus has the same relative handle decomposition. Moreover, it follows directly from the construction that $\mathcal{R}$ is a reference system for $w_{\mathfrak{S}}$ with $\mathfrak{S}$ as its twisted surface diagram.

In order to finish the proof of Theorem 1.1 we have to show that equivalent twisted surface diagrams give equivalent annular simple wrinkled fibrations. Recall that an equivalence of surface diagrams is a combination of two things: switching and a diffeomorphism. We will treat these separately.

Lemma 3.32. If $\mathfrak{S}$ and $\mathfrak{S}^{\prime}$ are diffeomorphic, then $w_{\mathfrak{S}}$ and $w_{\mathfrak{S}^{\prime}}$ are equivalent.

Proof. Let $\mathfrak{S}=(\Sigma, \Gamma, \mu), \mathfrak{S}^{\prime}=\left(\Sigma^{\prime}, \Gamma^{\prime}, \mu^{\prime}\right)$ and let $\phi: \Sigma \rightarrow \Sigma^{\prime}$ be a diffeomorphism such that $\Gamma^{\prime}=\phi(\Gamma)$ and $\mu^{\prime}=\phi \mu \phi^{-1}$. We will extend $\phi$ to a diffeomorphism $\hat{\phi}: W_{\mathfrak{S}} \rightarrow W_{\mathfrak{S}^{\prime}}$ which fits in the commutative diagram

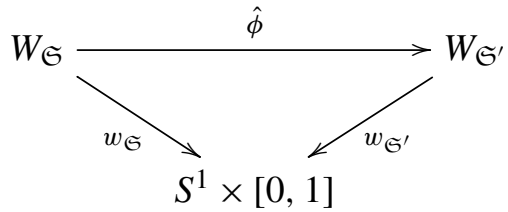


This will be done by going through the steps in the proof of Proposition 3.31. Let $W_{i}$ and $W_{i}^{\prime}, i=1,2$, denote the 4-manifolds built in each step.

From the identity $\mu^{\prime}=\phi \mu \phi^{-1}$ we deduce that $\phi$ induces a fiber-preserving diffeomorphism $\Sigma(\mu) \rightarrow \Sigma^{\prime}\left(\mu^{\prime}\right)$. Taking the product with the identity, we obtain $\hat{\phi}_{1}: W_{1} \rightarrow W_{1}^{\prime}$.

In the second step, where the 2-handles are attached to the curves in $\Gamma$, we simply note that $\hat{\phi}_{1}$ maps the attaching regions into each other and can thus be extended over the 2-handles to $\hat{\phi}_{2}: W_{2} \rightarrow W_{2}^{\prime}$. Note that the smoothing of the bumpy annulus does not cause any trouble since it does not involve the total space.

For the third step observe that, given a homotopy from $\left.w_{2}\right|_{\partial_{2} W_{2}}$ to a submersion, we can push it forward via $\left.\hat{\phi}_{2}\right|_{\partial_{2} W_{2}}$ to obtain such a homotopy for $\left.w_{2}^{\prime}\right|_{\partial_{2} W_{2}^{\prime}}$.

Lemma 3.33. If $\mathfrak{S}$ is a twisted surface diagram, then $w_{\mathfrak{S}}$ and $w_{\mathfrak{S}^{[1]}}$ are equivalent.

Proof. If we take the canonical reference system for $w_{\mathfrak{S}}$ and swing the last reference path once around the boundary, we obtain a reference system that induces $\mathfrak{S}^{[1]}$. Thus $w_{\mathfrak{S}}$ and $w_{\mathfrak{S}^{[1]}}$ are essentially the same annular simple wrinkled fibration.

3D. Gluing ambiguities. Recall that simple wrinkled fibrations over arbitrary base surfaces can be obtained from annular ones by gluing suitable surface bundles to the boundary components. To be precise, let $w_{0}: W \rightarrow A$ be an annular simple wrinkled fibration and let $\pi_{ \pm}: Y_{ \pm} \rightarrow B_{ \pm}$be surface bundles over surfaces $B_{ \pm}$such that there are boundary components $C_{ \pm} \subset B_{ \pm}$and fiber-preserving diffeomorphisms

$$
\psi_{ \pm}: \pi_{ \pm}^{-1}\left(C_{ \pm}\right) \rightarrow \partial_{ \pm} W .
$$

Then we can form a simple wrinkled fibration

$$
w: Y_{+} \cup_{\psi_{+}} W \cup_{\psi_{-}} Y_{-} \longrightarrow B_{+} \cup_{C_{+}} A \cup_{C_{-}} B_{-} .
$$

Of course, different choices of gluing diffeomorphisms may lead to inequivalent simple wrinkled fibrations. If we fix a pair $\psi_{ \pm}$of gluing maps, then we can obtain any other such pair by composing with automorphisms (in the sense of Section 2B) of the boundary fibrations $w_{0}: \partial_{ \pm} W \rightarrow S^{1}$. Obviously, isotopic gluing maps give rise to equivalent simple wrinkled fibrations and the gluing ambiguities are a priori parametrized by

$$
\pi_{0}\left(\operatorname{Aut}\left(\partial_{+} W, w\right)\right) \times \pi_{0}\left(\operatorname{Aut}\left(\partial_{-} W, w\right)\right) .
$$

However, it turns out that the first factor can be eliminated.

Lemma 3.34. Let $w: W \rightarrow A$ be an annular simple wrinkled fibration. Then any fiber-preserving diffeomorphism of $\partial_{+} W$ extends to a self-equivalence of $w$.

Proof. By Theorem 1.1 we can assume that $w$ is built from a twisted surface diagram $\mathfrak{S}=(\Sigma, \Gamma, \mu)$ such that $\partial_{+} W=\Sigma(\mu)$. According to (2-6) there are two 


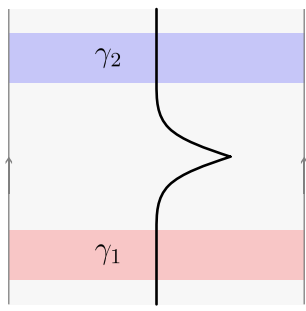

Figure 11. The relevant regions for extending nonconstant automorphisms.

types of automorphisms of $\Sigma(\mu)$, the constant ones coming from $C_{\operatorname{Mod}(\Sigma)}(\mu)$ and the nonconstant ones originating from $\pi_{1}(\operatorname{Diff}(\Sigma)$, id $)$. The statement that constant automorphisms of $\partial_{+} W$ extend to self-equivalences of $w$ is just a reformulation of Lemma 3.32. Thus it remains to treat the nonconstant ones.

By Theorem 2.10 these only occur when $\Sigma$ has genus one; we can thus assume that $\Sigma=T^{2}$. A refinement of Theorem 2.10 states that the map

$$
\pi_{1}\left(\operatorname{Diff}\left(T^{2}\right), \mathrm{id}\right) \rightarrow \pi_{1}\left(T^{2}, x\right),
$$

which sends an isotopy to the path traced out by a base point $x \in T^{2}$ during that isotopy, is an isomorphism; see [Earle and Eells 1969]. Note the first two curves in $\Gamma$, say $c_{1}$ and $c_{2}$, generate the fundamental group of $T^{2}$. Hence, we only have to extend the automorphisms coming from generators of $\pi_{1}\left(\operatorname{Diff}\left(T^{2}\right)\right.$, id) that map to $c_{1}$ and $c_{2}$ in (3-4). If we parametrize the torus by $S^{1} \times S^{1} \subset \mathbb{C}^{2}$ such that $S^{1} \times\{1\}$ maps to $c_{1}$ and $\{1\} \times S^{1}$ maps to $c_{2}$, then such generators are given by

$$
h_{t}^{c_{1}}(\xi, \eta):=\left(e^{2 \pi i t} \xi, \eta\right) \quad \text { and } \quad h_{t}^{c_{2}}(\xi, \eta):=\left(\xi, e^{2 \pi i t} \eta\right) \quad(t \in[0,1])
$$

and we denote the corresponding automorphisms of $\Sigma(\mu)$ by

$$
\varphi_{i}(x, t):=\left(h_{t}^{c_{i}}(x), t\right) .
$$

In order to extend $\varphi_{i}$ to $W_{\mathfrak{S}}$ we take one step back and homotope the path $h^{c_{i}}$ to be constant outside the interval where the 2-handle corresponding to $c_{i}$ is attached. These intervals (times $[0,1]$ ) are highlighted in Figure 11. Outside the preimage of the regions shown in Figure 11 we can simply extend $\varphi_{i}$ as the identity. In these region, observe that $h_{t}^{c_{i}}$ fixes $c_{i}$ setwise at all times, it just rotates it more and more as $t$ increases. It is easy to see that these rotations can be extended across the 2-handles in a way that respects the fibration structure.

Remark 3.35. The genus-one case of Example 3.7 shows that this Lemma does not hold in the absence of cusps. The above proof breaks down at the point where we need the vanishing cycles to generate the fundamental group. 


\section{Simple wrinkled fibrations over the disk and the sphere}

We now leave the general theory behind and focus on untwisted surface diagrams, that is, pairs $\mathfrak{S}=(\Sigma, \Gamma)$ where $\Gamma$ is a closed circuit in $\Sigma$, which we refer to simply as surface diagrams from now on. This will not lead to confusion since we will not encounter any twisted diagrams anymore.

By the results of the previous section, a surface diagram $\mathfrak{S}$ corresponds to an annular simple wrinkled fibration whose higher-genus boundary component has trivial monodromy. In fact, the higher-genus boundary of $w_{\mathfrak{S}}: W_{\mathfrak{S}} \rightarrow S^{1} \times[0,1]$ as constructed in Proposition 3.31 is canonically identified with the trivial fibration $\Sigma \times S^{1}$. We can thus fill this boundary component with $\Sigma \times D^{2}$ using some fiber-preserving diffeomorphism of $\Sigma \times S^{1}$ to obtain a simple wrinkled fibration over the disk. We denote the fibration obtain by gluing with the identity by $w_{\mathfrak{S}}: Z_{\mathfrak{S}} \rightarrow D^{2}$ or, by a slight abuse of notation, simply by $Z_{\mathfrak{S}}$ with the map to the disk implicitly understood. Since the boundary of the disk is contained in the lower-genus region, we refer to such fibrations as descending simple wrinkled fibrations (over the disk). According to Lemma 3.34, different gluing diffeomorphisms give rise to simple wrinkled fibrations equivalent to $Z_{\mathfrak{S}}$. We have thus established the following:

Proposition 4.1. There is a bijective correspondence between the respective equivalence classes of (untwisted) surface diagrams and descending simple wrinkled fibrations over the disk.

To make the connection to simple wrinkled fibrations over $S^{2}$, recall that by construction the boundary of $Z_{\mathfrak{S}}$ fibers over the circle. For the moment, let us say that $\mathfrak{S}$ has trivial monodromy if this boundary fibration is trivial (this will be made more precise in Definition 4.4 below). In this situation we can close off to a simple wrinkled fibration over $S^{2}$ by proceeding as above. More precisely, for a fixed boundary fiber $\Sigma^{\prime}$ in $Z_{\mathfrak{S}}$ we can choose a fiber-preserving diffeomorphism $\varphi: \Sigma^{\prime} \times S^{1} \rightarrow \partial Z_{\mathfrak{S}}$ and fill the boundary of $Z_{\mathfrak{S}}$ with a copy of $\Sigma^{\prime} \times D^{2}$. The result is a closed 4-manifold $X_{\mathfrak{S}}^{\varphi}=Z_{\mathfrak{S}} \cup_{\varphi} \Sigma^{\prime} \times D^{2}$ equipped with a simple wrinkled fibration over $S^{2}$ which we denote by $w_{\mathfrak{S}}^{\varphi}$. Unfortunately, this gluing process is more delicate. The main problem is that there is no canonical choice for $\varphi$; moreover, if the genus of $\Sigma^{\prime}$ is low, then different choices can lead to inequivalent fibrations. Combining Proposition 4.1 with the discussion in Section 3D and Theorem 2.10 leads to the cleanest possible statement:

Corollary 4.2. Let $g>0$ be a positive integer.

(1) For $g \geq 3$ there is a one-to-one correspondence between equivalence classes of genus $g$ surface diagrams with trivial monodromy and genus $g$ simple wrinkled fibrations over $S^{2}$. 
(2) For $g=2$ (respectively $g=1$ ) the set of equivalence classes of genus $g$ simple wrinkled fibrations over $S^{2}$ with equivalent surface diagrams admits a transitive action of $\mathbb{Z} \oplus \mathbb{Z}$ (respectively $\mathbb{Z}_{2}$ ).

Recall that, according to Theorem 3.8, we can obtain all closed, oriented, smooth 4-manifolds from surface diagrams by the above process. It is thus of great interest to understand which surface diagrams have trivial monodromy and actually describe closed 4-manifolds. The following example indicates that most surface diagrams will not have trivial monodromy.

Example 4.3. Let $\Sigma$ be a closed, orientable surface together with a mapping class $\phi \in \operatorname{Mod}(\Sigma)$. Then any factorization of $\mu$ into positive Dehn twists yields a Lefschetz fibration over the disk whose boundary can be identified with the mapping torus $\Sigma(\phi)=(\Sigma \times[0,1]) /(x, 1) \sim(\phi(x), 0)$. As in Example 3.6 we can turn this Lefschetz fibration into a descending simple wrinkled fibration without changing the boundary. Thus any surface bundle over the circle (with closed fibers) bounds some descending simple wrinkled fibration over the disk and any mapping class can be realized as the monodromy of a surface diagram.

In fact, the situation is very similar to the theory of Lefschetz fibrations. Any word in positive Dehn twists (or, equivalently, a finite sequence of simple closed curves) on a closed, oriented surface determines a Lefschetz fibration over the disk, the boundary fibers over the circle with monodromy being given by the product of the Dehn twists; and if this monodromy is trivial, one can close off to a Lefschetz fibration over $S^{2}$. Just as an arbitrary product of Dehn twists will not be isotopic to the identity, so will a surface diagram not give rise to a simple wrinkled fibration over $S^{2}$. The advantage of the Lefschetz setting is the direct control over the boundary.

4A. The monodromy of a surface diagram. In order to obtain a more intrinsic description of the boundary of $Z_{\mathfrak{S}}$ in terms of $\mathfrak{S}$ we need a little detour. Let $a, b \subset \Sigma$ be a pair of simple closed curves in a surface $\Sigma$ that intersect transversely in a single point. We denote by $\Sigma_{a}$ and $\Sigma_{b}$ the surfaces obtained by surgery on the curves $a$ and $b$, respectively. To be concrete, we fix tubular neighborhoods $v a$ and $v b$ and consider $\Sigma_{a}$ (respectively $\Sigma_{b}$ ) as the result of filling in the two boundary components of $\Sigma \backslash v a$ (respectively $\Sigma \backslash v b$ ) with disks. We can assume that $v(a \cup b):=v a \cup v b$ is diffeomorphic to a once punctured torus - for convenience we also assume that it has a smooth boundary in $\Sigma$. Observe that $\Sigma \backslash v(a \cup b)$ has one boundary component and is contained in both $\Sigma_{a}$ and $\Sigma_{b}$ as a subsurface. Furthermore, the closure of $v b \backslash v a$ (respectively $v a \backslash v b$ ) is a disk in $\Sigma_{a}$ (respectively $\Sigma_{b}$ ). It follows that, up to isotopy, there is a unique diffeomorphism

$$
\kappa_{a, b}: \Sigma_{a} \rightarrow \Sigma_{b}
$$


which restricts to the identity on $\Sigma \backslash v(a \cup b)$. Furthermore, we can assume that $\kappa_{a, b}$ maps $b \backslash v a$ onto $a \backslash v b$.

Now let $\mathfrak{S}=\left(\Sigma ; c_{1}, \ldots, c_{l}\right)$ be a surface diagram and consider the associated simple wrinkled fibration $w_{\mathfrak{S}}: Z_{\mathfrak{S}} \rightarrow D^{2}$. Then each adjacent pair of curves $c_{i}$ and $c_{i+1}$ fits the above situation and we thus get a collection of diffeomorphisms

$$
\kappa_{c_{i}, c_{i+1}}: \Sigma_{c_{i}} \rightarrow \Sigma_{c_{i+1}} .
$$

Moreover, it follows from the definition of surface diagrams that the composition

$$
\mu_{\mathfrak{S}}:=\kappa_{c_{l}, c_{1}} \circ \kappa_{c_{l-1}, c_{l}} \circ \cdots \circ \kappa_{c_{1}, c_{2}}
$$

maps $\Sigma_{c_{1}}$ to itself and it is easy to see that its isotopy class does not depend on any of the implicit choices involved in its definition.

Definition 4.4. The mapping class $\mu_{\mathfrak{S}} \in \operatorname{Mod}\left(\Sigma_{c_{1}}\right)$ represented by the composition above is called the monodromy of $\mathfrak{S}$.

This name is justified by the following lemma.

Lemma 4.5. Let $\mathfrak{S}=(\Sigma, \Gamma)$ be a surface diagram. Then the boundary fibration $\left(\partial Z_{\mathfrak{S}}, w_{\mathfrak{S}}\right)$ can be identified with the mapping torus $\Sigma_{c_{1}}\left(\mu_{\mathfrak{S}}\right)$.

Proof. By the construction of $w_{\mathfrak{S}}$ its fiber over the origin is naturally identified with $\Sigma$. Furthermore, recall that the annular fibration associated to $\mathfrak{S}$ is equipped with a reference system whose reference paths we can naturally extend from the annulus to the disk by connecting them to the origin. The result is a collection of reference paths $R_{1}, \ldots, R_{l}$ from the origin to the boundary of the disk and we denote its endpoints by $\theta_{1} \ldots, \theta_{l} \in S^{1}$. Observe that such a reference path, say $R_{i}$, gives rise to an identification of the fiber over $\theta_{i}$ with the surface $\Sigma_{c_{i}}$ obtained from surgery on $c_{i}$ where $c_{i}$ is the vanishing cycle associated to $R_{i}$.

Now consider the region in the base bounded by two adjacent reference paths $R_{i}$ and $R_{i+1}$. Using a suitable notion of parallel transport we see that the preimage of this region contains a trivial bundle with fiber $\Sigma \backslash v\left(c_{i} \cup c_{i+1}\right)$. In particular, the parallel transport along the boundary segment from $\theta_{i}$ to $\theta_{i+1}$ restricts to the identity on the complement of $v\left(c_{i} \cup c_{i+1}\right)$ and thus must be isotopic to $\kappa_{c_{i}, c_{i+1}}$ and the claim follows.

It is also possible to describe the monodromy in terms of the original surface $\Sigma$. This takes us on another small detour. Let $a \subset \Sigma$ be a nonseparating simple closed curve in a surface $\Sigma$ and let $\operatorname{Mod}(\Sigma, a)$ denote the subgroup of $\operatorname{Mod}(\Sigma)$ consisting of all elements that fix $a$ up to isotopy. Recall that there is a short exact sequence

$$
1 \longrightarrow\left\langle\tau_{a}\right\rangle \longrightarrow \operatorname{Mod}(\Sigma, a) \stackrel{\operatorname{cut}_{a}}{\longrightarrow} \operatorname{Mod}(\Sigma \backslash a) \longrightarrow 1
$$


where we consider $\Sigma \backslash a$ as a twice punctured surface (see [Farb and Margalit 2011, Chapter 3] and also [Ivanov 1992, Section 7.5] for a proof that cut ${ }_{a}$ is well defined). The complement $\Sigma \backslash a$ can be related to the surgered surface $\Sigma_{a}$ as follows. In $\Sigma_{a}$ there is an obvious pair of points, namely the centers of the surgery disks. If we denote by $\Sigma_{a}^{*}$ the surface obtained by marking these points, then $\Sigma \backslash a$ is canonically identified (at least up to isotopy) with $\Sigma_{a}^{*}$ and thus $\operatorname{Mod}(\Sigma \backslash a)$ is canonically isomorphic to $\operatorname{Mod}\left(\Sigma_{a}^{*}\right)$. Hence, we can define the surgery homomorphism

$$
\Phi_{a}: \operatorname{Mod}(\Sigma, a) \rightarrow \operatorname{Mod}\left(\Sigma_{a}\right)
$$

as the composition

$$
\operatorname{Mod}(\Sigma, a) \underset{\operatorname{cut}_{a}}{\longrightarrow} \operatorname{Mod}(\Sigma \backslash a) \underset{\cong}{\longrightarrow} \operatorname{Mod}\left(\Sigma_{a}^{*}\right) \underset{\text { forget }}{\longrightarrow} \operatorname{Mod}\left(\Sigma_{a}\right)
$$

where the last map is induced by forgetting the marked points in $\Sigma_{a}^{*}$.

Applying this to surface diagram we obtain the following.

Lemma 4.6. Let $\mathfrak{S}=\left(\Sigma ; c_{1}, \ldots, c_{l}\right)$ be a surface diagram. Then

$$
\tilde{\mu}_{\mathfrak{S}}:=\tau_{\tau_{c_{l}}\left(c_{1}\right)} \circ \tau_{\tau_{c_{l-1}}\left(c_{l}\right)} \circ \tau_{\tau_{c_{1}}\left(c_{2}\right)} \in \operatorname{Mod}(\Sigma)
$$

is contained in $\operatorname{Mod}\left(\Sigma, c_{1}\right)$ and satisfies $\Phi_{c_{1}}\left(\tilde{\mu}_{\mathfrak{S}}\right)=\mu_{\mathfrak{S}}$.

Proof. Since $c_{i}$ and $c_{i+1}$ are geometrically dual, the mapping class $\tau_{\tau_{c_{l-1}}\left(c_{l}\right)}$ has a representative $T \in \operatorname{Diff}^{+}(\Sigma)$ that maps $c_{i}$ to $c_{i+1}$ (as a set). The claim then follows from the observation that the diagram

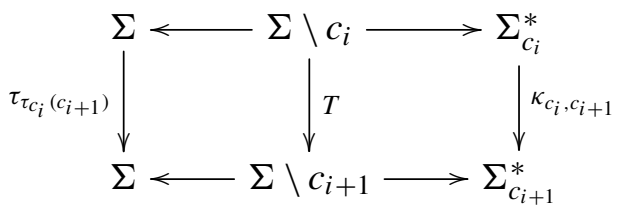

commutes up to isotopy.

The above makes it interesting to study the map $\Phi_{c_{1}}$ and its kernel.

Lemma 4.7. Let $a \subset \Sigma$ be a nonseparating simple closed curve. The group $\operatorname{Mod}(\Sigma, a)$ is generated by elements of the form $\tau_{c}$ and $\Delta_{a, b}:=\left(\tau_{a} \tau_{b}\right)^{3}$, where $i(a, c)=0$ and $i(a, b)=1$.

We refer to the mapping classes $\Delta_{a, b}$ as $\Delta$-twists. Note that $\Delta$-twists are defined for arbitrary pairs of geometrically dual curves and do not have to involve the curve $a$ in the above Lemma.

Proof. It follows from the short exact sequence (4-1) that we can obtain a generating set for $\operatorname{Mod}(\Sigma, a)$ by lifting a generating set for $\operatorname{Mod}(\Sigma \backslash a)$ and adding the Dehn 
twist about $a$. As a generating set for $\operatorname{Mod}(\Sigma \backslash a)$ we can take the collection Dehn twists and so called half-twists about simple arcs connecting the two punctures. Then the Dehn twists in $\operatorname{Mod}(\Sigma \backslash a)$ have obvious lifts in $\operatorname{Mod}(\Sigma)$ and it is easy to see that each half-twist lifts to a $\Delta$-twist.

Corollary 4.8. The kernel of $\Phi_{a}: \operatorname{Mod}(S, a) \rightarrow \operatorname{Mod}\left(\Sigma_{a}\right)$ contains the Dehn twist $\tau_{a}$ as well as all $\Delta$-twists involving a.

The expert will have noticed that the mapping class $\tilde{\mu}_{\mathfrak{S}}$ in Lemma 4.6 is simply the monodromy of the boundary of the Lefschetz part of the simplified broken Lefschetz fibration obtained from $w_{\mathfrak{S}}$ by unsinking all the cusps. Of course, there are many different lifts of $\mu_{\mathfrak{S}}$ to $\operatorname{Mod}(\Sigma)$. For example, it follows from the braid relations for the pairs of adjacent curves that

$$
\begin{aligned}
\tilde{\mu}_{\mathfrak{S}} & =\tau_{c_{1}}^{-c}\left(\tau_{c_{l}} \tau_{c_{1}}\right)\left(\tau_{c_{l-1}} \tau_{c_{l}}\right) \ldots\left(\tau_{c_{1}} \tau_{c_{2}}\right) \\
& =\tau_{c_{1}}^{-2 c}\left(\tau_{c_{l}} \tau_{c_{1}} \tau_{c_{l}}\right)\left(\tau_{c_{l-1}} \tau_{c_{l}} \tau_{c_{l-1}}\right) \ldots\left(\tau_{c_{1}} \tau_{c_{2}} \tau_{c_{1}}\right)
\end{aligned}
$$

and since $\tau_{c_{1}}$ is contained in the kernel of $\Phi_{c_{1}}$ we obtain two other choices. To illustrate these mapping class group techniques we produce some examples of surface diagrams with trivial monodromy.

Example 4.9. Given a not necessarily closed circuit $\Gamma=\left(c_{1}, \ldots, c_{l}\right)$ in an oriented surface $\Sigma$ we can form a closed circuit $D \Gamma:=\left(c_{1}, \ldots, c_{l-1}, c_{l}, c_{l-1}, \ldots, c_{2}\right)$ which we call the double of $\Gamma$. We claim that the surface diagram $D \mathfrak{S}:=(\Sigma, D \Gamma)$ has trivial monodromy. For convenience let us write $\tau_{i}=\tau_{c_{i}}$. As explained above the monodromy of $D \mathfrak{S}$ can be lifted to $\operatorname{Mod}(\Sigma)$ as

$$
\begin{aligned}
\mu & =\left(\tau_{2} \tau_{1} \tau_{2}\right) \ldots\left(\tau_{l-2} \tau_{l-1} \tau_{l-2}\right)\left(\tau_{l-1} \tau_{l} \tau_{l-1}\right)\left(\tau_{l} \tau_{l-1} \tau_{l}\right)\left(\tau_{l-1} \tau_{l-2} \tau_{l-1}\right) \ldots\left(\tau_{1} \tau_{2} \tau_{1}\right) \\
& =\left(\tau_{2} \tau_{1} \tau_{2}\right) \ldots\left(\tau_{l-2} \tau_{l-1} \tau_{l-2}\right) \Delta_{c_{l-1}, c_{l}}\left(\tau_{l-1} \tau_{l-2} \tau_{l-1}\right) \ldots\left(\tau_{1} \tau_{2} \tau_{1}\right) .
\end{aligned}
$$

Our goal is to factor this expression into a sequence of $\Delta$-twists involving $c_{1}$. The key observation is that

$$
\begin{aligned}
& \left(\tau_{l-2} \tau_{l-1} \tau_{l-2}\right) \Delta_{c_{l-1}, c_{l}}\left(\tau_{l-1} \tau_{l-2} \tau_{l-1}\right) \\
& =\left(\tau_{l-2} \tau_{l-1} \tau_{l-2}\right) \Delta_{c_{l-1}, c_{l}}\left(\tau_{l-2} \tau_{l-1} \tau_{l-2}\right) \\
& =\left(\tau_{l-2} \tau_{l-1} \tau_{l-2}\right) \Delta_{c_{l-1}, c_{l}}\left(\tau_{l-2} \tau_{l-1} \tau_{l-2}\right)^{-1} \Delta_{c_{l-2}, c_{l-1}} \\
& =\Delta_{\tau_{l-2} \tau_{l-1} \tau_{l-2}\left(c_{l-1}\right), \tau_{l-2} \tau_{l-1} \tau_{l-2}\left(c_{l}\right)} \Delta_{c_{l-2}, c_{l-1}} \\
& =\Delta_{c_{l-2}, \tau_{l-2} \tau_{l-1} \tau_{l-2}\left(c_{l}\right)} \Delta_{c_{l-2}, c_{l-1}} \text {. }
\end{aligned}
$$

Applying this repeatedly we eventually obtain

$$
\mu=\Delta_{c_{1}, \delta_{l}} \Delta_{c_{1}, \delta_{l-1}} \ldots \Delta_{c_{1}, \delta_{2}}
$$


where $\delta_{k}:=\tau_{1} \tau_{2} \tau_{1} \ldots \tau_{k-2} \tau_{k-1} \tau_{k-2}\left(c_{k}\right)$. Hence, the monodromy of $D \mathfrak{S}$ is trivial by Corollary 4.8 .

It is also possible to show that $D \mathfrak{S}$ has trivial monodromy by directly constructing a simple wrinkled fibration over $S^{2}$. This construction will also justify our terminology. The key observation is that, even if $\Gamma$ is not closed, the ideas in the proof of Proposition 3.31 can be used to build a wrinkled fibration over the disk.

Indeed, by attaching 2-handles to $\Sigma \times D^{2}$ along the fiber framed curves $c_{i}$ in boundary fibers ordered according to the orientation of $S^{1}$ we obtain a 4-manifold with boundary $P_{\Gamma}$ together with a map to the disk which has an arc of folds for each 2-handle and each arc gives rise to a pair of Morse critical points on the boundary. As in the third step of the proof of Proposition 3.31 we can trade pairs of critical points on the boundary coming from $c_{i}$ and $c_{i+1}, i<l$, for cusps in the interior. What remains is a wrinkled fibration on $P_{\Gamma}$ over the disk with two critical points on the boundary, one coming from $c_{1}$ and the other from $c_{l}$. Of course, if $\Gamma$ is closed, then $P_{\Gamma}$ is diffeomorphic to $Z_{\mathfrak{S}}$ where $\mathfrak{S}=(\Sigma, \Gamma)$, but the corresponding map to the disk is different.

If we apply this construction to the reversed circuit $\bar{\Gamma}=\left(c_{l}, \ldots, c_{1}\right)$, then we obtain another 4-manifold $P_{\bar{\Gamma}}$ and it is easy to see that the self-diffeomorphism of $\Sigma \times D^{2}$ which sends $(p, x)$ to $(p,-x)$ induces an orientation-preserving diffeomorphism from $P_{\bar{\Gamma}}$ to $\bar{P}_{\Gamma}$. We thus obtain a wrinkled fibration on $\bar{P}_{\Gamma}$ and the identity map of $\partial P_{\Gamma}$ provides an orientation-reversing and fiber-preserving diffeomorphism of the boundary fibrations on $P_{\Gamma}$ and $\bar{P}_{\Gamma}$. Hence, the fibrations on $P_{\Gamma}$ and $\bar{P}_{\Gamma}$ give rise to a wrinkled fibration over $S^{2}$ on the double $D P_{\Gamma}=P_{\Gamma} \cup_{\text {id }} \bar{P}_{\Gamma}$ which turns out to be a simple wrinkled fibration with surface diagram $D \mathfrak{S}$.

4B. Drawing Kirby diagrams. In this section we show how to translate surface diagrams into Kirby diagrams of the associated simple wrinkled fibrations. For the necessary background we refer the reader to [Gompf and Stipsicz 1999]. Throughout, we use Akbulut's dotted circle notation for 1-handles to avoid ambiguities for framing coefficients.

Descending simple wrinkled fibrations. Let $w_{\mathfrak{S}}: Z_{\mathfrak{S}} \rightarrow D^{2}$ be a descending simple wrinkled fibration of genus $g$ with surface diagram $\mathfrak{S}=\left(\Sigma_{g} ; c_{1}, \ldots, c_{l}\right)$. Recall that the associated handle decomposition of $Z$ is obtained from (some handle decomposition of) $\Sigma_{g} \times D^{2}$ by attaching 2-handles along $c_{i} \subset \Sigma_{g} \times\left\{\theta_{i}\right\}$ with the fiber framing where $\theta_{1}, \ldots, \theta_{l} \in S^{1}$ are ordered according to the orientation on $S^{1}$. So in order to draw a Kirby diagram for $Z_{\mathfrak{S}}$ we need to find a diagram for $\Sigma \times D^{2}$ in which the fibers of the boundary should be as clearly visible as possible.

A convenient choice is the diagram shown in Figure 12 which is induced from the obvious handle decomposition of $\Sigma_{g}$ with one 0-handle, $2 g$ 1-handles and one 2-handle. One fiber of $\Sigma_{g} \times S^{1}$, which we identify with $\Sigma_{g}$, is clearly visible and 


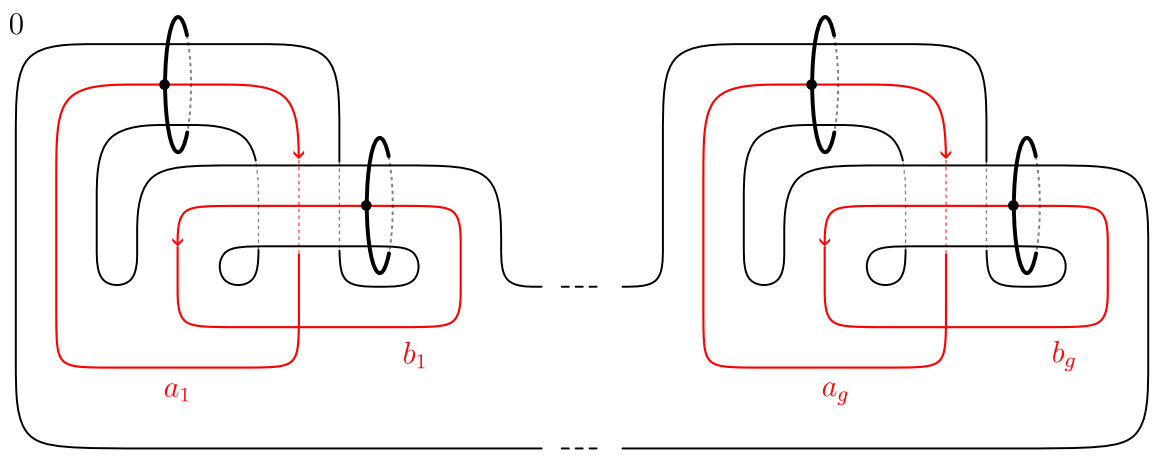

Figure 12. A diagram for $\Sigma_{g} \times D^{2}$ where fiber and blackboard framing agree. The red curves show a basis for $H_{1}\left(\Sigma_{g}\right)$.

the canonical generators $a_{1}, b_{1}, \ldots, a_{g}, b_{g}$ for $H_{1}\left(\Sigma_{g}\right)$ are also indicated. We have chosen the orientations such that $\left\langle a_{i}, b_{i}\right\rangle_{\Sigma_{g}}=1$. Another advantage of this picture is that the fiber framing agrees with the blackboard framing. One minor drawback is that the picture does not immediately show all fibers of $\Sigma_{g} \times S^{1}$ but only an interval worth of them (just thicken the surface a little). However, this is actually enough for our purposes since we only need the fibers over the interval $\left[\theta_{1}, \theta_{l}\right] \subset S^{1}$. To get the orientations right we require that the orientation of the fiber agrees with the standard orientation of the plane and, according to the "fiber first convention", the positive $S^{1}$-direction points toward the reader.

With this understood, it is easy to locate the attaching curves of the fold handles in the diagram and it remains to determine their framing coefficients. More generally, we can describe the linking form of the link corresponding to the fold handles. It should be no surprise that the framing and linking information in the diagram depends on our choice of the handle decomposition for $\Sigma_{g}$.

Let $c \subset \Sigma_{g}$ be a simple closed curve. After choosing an orientation its homology class $[c] \in H_{1}(\Sigma)$ can be expressed as

$$
[c]=\sum_{i=1}^{g}\left(n_{a_{i}}(c) a_{i}+n_{b_{i}}(c) b_{i}\right) .
$$

We identify $\Sigma_{g}$ with $\Sigma_{g} \times\{0\}$ and, by a slight abuse of notation, we continue to denote the canonical push-off of $c$ to $\Sigma_{g} \times\{z\}, z \in D^{2}$, by $c$.

Lemma 4.10. For a simple closed curve $c \subset \Sigma_{g} \times\{\theta\}, \theta \in\left[\theta_{1}, \theta_{l}\right] \subset S^{1}$, the framing coefficient of the fiber framing in Figure 12 is given by

$$
\operatorname{fr}(c)=\sum_{i=1}^{g} n_{a_{i}}(c) n_{b_{i}}(c) .
$$




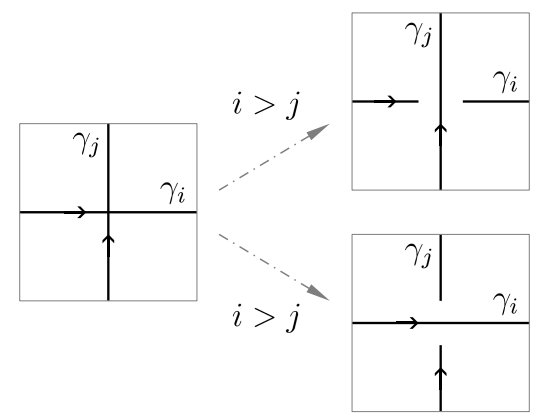

Figure 13. An intersection in a surface diagram and its crossing in the Kirby diagram.

Furthermore, if $c \subset \Sigma_{g} \times\{\theta\}$ and $c^{\prime} \subset \Sigma_{g} \times\left\{\theta^{\prime}\right\}$ are two oriented simple closed curves, with $\theta, \theta^{\prime} \in\left[\theta_{1}, \theta_{l}\right]$, their linking number in Figure 12 is

$$
\operatorname{lk}\left(c, c^{\prime}\right)=\frac{1}{2} \operatorname{sgn}\left(\theta-\theta^{\prime}\right)\left\langle c, c^{\prime}\right\rangle+\frac{1}{2} \sum_{i=1}^{g}\left(n_{a_{i}}(c) n_{b_{i}}\left(c^{\prime}\right)+n_{a_{i}}\left(c^{\prime}\right) n_{b_{i}}(c)\right),
$$

where $\left\langle c, c^{\prime}\right\rangle$ is the algebraic intersection number of $c$ and $c^{\prime}$ in $\Sigma_{g}$ and $\operatorname{sgn}$ denotes the sign of a real number.

Proof. First observe that $c \subset \Sigma_{g} \times\{\theta\}$ can be isotoped off the 2-handle of $\Sigma_{g}$ so that it becomes completely visible in Figure 12 and, since the fiber framing and blackboard framing agree, its framing coefficient is given by its writhe in the diagram - the signed count of crossings with some chosen orientation. From the way the diagram is drawn it is clear that each crossing is caused by $c$ running over $a_{i}$ and $b_{i}$ for some $i$ and that their signed sum is given by the right side of (4-2).

The statement about linking numbers follows from a similar count of crossings. Recall that the linking number of two oriented knots can be computed from any link diagram as half of the signed number of crossings. The second term on the right side of (4-3) arises just as above. However, the first term deserves some explanation. Each (transverse) intersection point of $c$ and $c^{\prime}$ in $\Sigma_{g}$ contributes a crossing in the diagram. Now, the sign of the crossing depends on two things: the sign of the intersection point and the information which strand is on top in the diagram. From Figure 13 we see that the contribution of each crossing is exactly as in (4-2).

Remark 4.11. Formula (4-3) can be used to obtain a description of the intersection form of the 4-manifold $Z_{\mathfrak{S}}$ using only the data in $\mathfrak{S}$. Also, since (4-3) only depends on the homology classes of the curves in $\mathfrak{S}$, so do the intersection form and, in particular, the signature of $Z_{\mathfrak{S}}$. We will return to this observation in a future publication.

The diagrams of simple wrinkled fibrations derived from Figure 12 are good for abstract reasoning, however, in practice it is convenient to start with a cleaner 


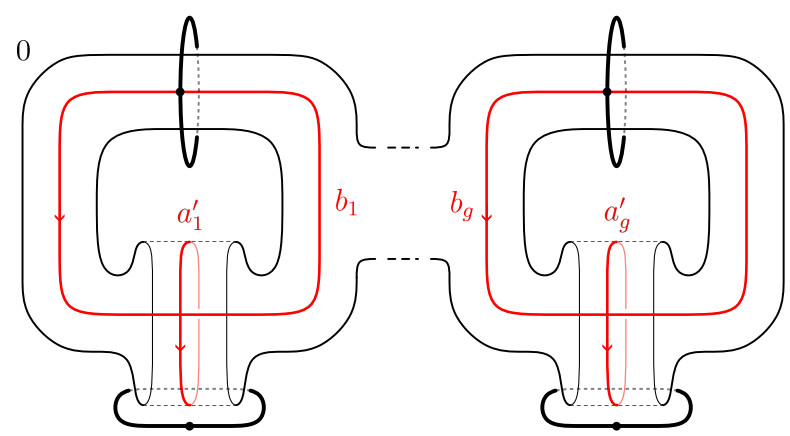

Figure 14. A cleaner diagram of $\Sigma_{g} \times D^{2}$.

diagram for $\Sigma_{g} \times D^{2}$ such as the one shown in Figure 14. In this picture, the fiber appears as the boundary sum of regular neighborhoods of the basis curves $\left\{a_{i}^{\prime}, b_{i}\right\}_{i=1}^{g}$ which, in turn, appear as meridians to the dotted circles. The framing coefficient of the fiber framing for simple closed curves on a fiber in Figure 14 can be computed as follows. It is not hard to see that Figure 14 is obtained from Figure 12 by a sequence of 1-handle slides and an isotopy of the 2-handle and vice versa. Note that these moves do not change the framing coefficients of any other 2-handles that might have been around. Moreover, during the moves, the $b$-curves remain fixed, while the $a$-curves undergo some changes. When pulling $a_{i}^{\prime}$ in Figure 14 back to Figure 12 one obtains a representative for the element

$$
\left[a_{1}, b_{1}\right] * \cdots *\left[a_{i-1}, b_{i-1}\right] * a_{i} \in \pi_{1}\left(\Sigma_{g}\right)
$$

where $[x, y]=x y x^{-1} y^{-1}$. The important observation is that while this curve is not isotopic to $a_{i}$ it does represent the same homology class. As a consequence, formula (4-2) can be used for Figure 14 with $a_{i}$ replaced by $a_{i}^{\prime}$.

Closing off and the last 2-handle. Recall that our motivation comes from Williams' theorem that all closed, oriented 4-manifolds admit simple wrinkled fibrations over $S^{2}$. We have seen that these can be described (up to equivalence) by surface diagrams with trivial monodromy and we have already mentioned that it is in general not easy to check whether the monodromy of a given surface diagram is trivial. But the situation is even worse. Say that we know for some reason that a given surface diagram has trivial monodromy and let us also assume that the genus is at least three so that there are no gluing ambiguities. Even in this case it is not clear at all how the surface diagram encodes the information to complete the Kirby diagram.

To be more precise, let $w: X \rightarrow S^{2}$ be a simple wrinkled fibration with surface diagram S. Let $v \Sigma_{-}$be a neighborhood of a lower-genus fiber and let $Z:=X \backslash v \Sigma_{-}$. Then $w$ restricts to a descending simple wrinkled fibration on $Z$ and $\partial Z$ can be identified with $\Sigma_{-} \times S^{1}$ so that $\mathfrak{S}$ must have trivial monodromy. We can draw a 
Kirby diagram for $Z$ as described in the previous section and to complete it to a diagram for $X$ we have to understand how to glue $\nu \Sigma_{-}$back in.

We can choose a handle decomposition for $v \Sigma_{-}$with one 0 -handle, $2 g\left(\Sigma_{-}\right)$ 1-handles and one 2-handle. Turning this upside down results in a relative handle decomposition on $\partial Z \cong \Sigma_{-} \times S^{1}$ with one 2-handle, $2 g\left(\Sigma_{-}\right)$3-handles and a 4-handle. The general theory tells us that the 3- and 4-handles attach in a standard way once we know how to attach the 2-handle. Unfortunately, it turns out to be rather difficult to locate this last 2-handle in the Kirby diagram for $Z$.

Our knowledge about the last 2-handle is a priori limited to the following observation. If we identify $v \Sigma_{-}$with $\Sigma_{-} \times D^{2}$, then the attaching curve of the last 2-handle corresponds to $\{p\} \times \partial D^{2}$ for some $p \in \Sigma_{-}$. In particular, we see that it must be attached along a section of the boundary fibration $(\partial Z, w)$.

Remark 4.12. Given a surface diagram $\mathfrak{S}$ with trivial monodromy there is a general method for finding possible last 2-handles for $Z_{\mathfrak{S}}$ which is not very conceptual but still useful in some situations. One considers a Kirby diagram for $Z_{\mathfrak{S}}$ as a surgery diagram for $\partial Z_{\mathfrak{S}}$ and performs (3-dimensional) Kirby moves until the fibration structure is clearly visible as $\Sigma_{-} \times S^{1}$. In such a diagram it is easy to locate attaching curves for possible last 2-handles which one can then pull back to the original diagram by undoing the moves and dragging the curves along. This strategy also works for Lefschetz fibrations as discussed in [Gompf and Stipsicz 1999, Chapter 8.2].

Just as in the Lefschetz case, the situation becomes easier if one knows that $Z_{\mathfrak{S}}$ can be closed off to a fibration over $S^{2}$ which admits a section. The proof of the following lemma is the same as in the Lefschetz case and we refer the reader to [Gompf and Stipsicz 1999].

Lemma 4.13. Let $w: X \rightarrow S^{2}$ be a simple wrinkled fibration with surface diagram $\mathfrak{S}$. If $w$ admits a section of self-intersection $k$, then the last two handle appears in the diagram for $Z_{\mathfrak{S}}$ as a $k$-framed meridian of the 2-handle corresponding to the fiber. Furthermore, if $\mathfrak{S}$ is a surface diagram and a meridian as above can be used to attach the last 2-handle, then the corresponding simple wrinkled fibration admits a section of self-intersection $k$.

In order to illustrate Remark 4.12 and Lemma 4.13 as well as our method of drawing Kirby diagrams we give an example which is also a warm-up for the next section.

Example 4.14. Let $a, b \subset \Sigma_{g}$ be a geometrically dual pair of simple closed curves. We claim that $\mathfrak{S}=\left(\Sigma_{g} ; a, \tau_{b}(a), b\right)$ is a surface diagram for $\Sigma_{g-1} \times S^{2} \# \overline{\mathbb{C} P^{2}}$. We can assume that $a$ and $b$ are the standard generators $a_{1}$ and $b_{1}$ in Figure 14 and Figure 15 shows the final Kirby diagram. In order to see how we got there let us first 


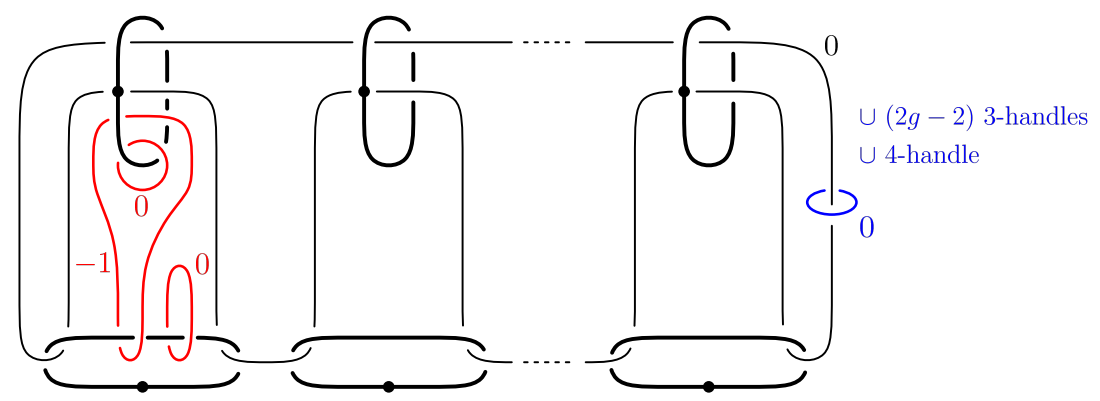

Figure 15. Manifolds with surface diagram $\left(\Sigma_{g} ; a, \tau_{b}(a), b\right)$.

ignore all the blue components. What is left is just the Kirby diagram for $Z_{\mathfrak{S}}$. The framings on the fold handles can either be computed using Lemma 4.10 (together with Proposition 2.7) or by hand (the curve is simple enough to draw a parallel push-off in the fiber direction and compute the linking number). We now perform the obvious handle moves: using the meridians to the two 1-handles on the left we first unlink the -1 -framed fold handle (corresponding to $\tau_{b}(a)$ ) to obtain a -1 -framed unknot isolated from the rest of the diagram, then we unlink the black 2-handle (corresponding to the fiber) and finally cancel the 1-handles and their meridians. Obviously, the thus obtained diagram shows $\Sigma_{g-1} \times D^{2} \# \overline{\mathbb{C} P^{2}}$ and the boundary is clearly visible as $\Sigma_{g-1} \times S^{1}$. Moreover, it is easy to see that the last 2-handle can be attached along a 0 -framed meridian to the fiber 2-handle and the resulting manifold is $\Sigma_{g-1} \times S^{2} \# \overline{\mathbb{C} P^{2}}$ as claimed. Finally, since we attached the last 2-handle in a region that was not affected by the Kirby moves it will not change when we undo the moves again and we arrive at Figure 15. Lemma 4.13 then tells us that the corresponding simple wrinkled fibration will have a section of self-intersection zero.

Note that for $g \geq 3$ any other choice for the last 2-handle that might have been possible leads to an equivalent fibration whose total space is diffeomorphic to $\Sigma_{g-1} \times S^{2} \# \overline{\mathbb{C} P^{2}}$. In the lower-genus cases there are more options. However, in any case one will end up with a blow-up of some surface bundle over $S^{2}$.

4C. Relation to broken Lefschetz fibrations. Let $w: X \rightarrow B$ be a simple wrinkled fibration. After trading all the cusps for Lefschetz singularities by applying Lekili's unsinking modification we obtain a broken Lefschetz fibration

$$
\beta_{w}: X \rightarrow B
$$

with one round singularity, smoothly embedded in the base, and all its Lefschetz points on the higher-genus side. If the base is the sphere or the disk, then $\beta_{w}$ is a simplified broken Lefschetz fibration in the sense of [Baykur 2009] and thus induces another handle decomposition of $X$. 
In order to relate these two handle decompositions, let us briefly review how a simplified broken Lefschetz fibration $\beta: X \rightarrow B$ gives rise to a handle decompositions. Much in the spirit of simple wrinkled fibrations one chooses a reference point in the higher-genus region together with a collection of disjointly embedded arcs $L_{1}, \ldots, L_{k}, R \subset B$, where $k$ is the number of Lefschetz singularities, emanating from the reference point such that each $L_{i}$ ends in a Lefschetz point and $R$ passes through the round singularity once. Such a system of arcs is known as a Hurwitz system for $\beta$. The arcs in a Hurwitz system then give rise to simple closed curves in the reference fiber $\Sigma$ to which we shall refer to as the Lefschetz vanishing cycles $\lambda_{1}, \ldots, \lambda_{k} \subset \Sigma$ and the round vanishing cycle $\rho$. A handle decomposition of $X$ is then given as follows:

- Start with $\Sigma \times D^{2}$.

- Going around $S^{1}$, attach a Lefschetz handle along the $\lambda_{i}$ pushed off into fibers over $S^{1}$ (that is, 2-handles with framing -1 with respect to the fiber framing).

- Attach a round 2-handle along $\rho$.

The round 2-handle decomposes into a 2-handle and a 3-handle such that the 3handle goes over the 2-handle geometrically twice and the 2-handle is attached along $\rho$ with respect to the fiber framing. (For more details see [Baykur 2009].)

Now let $w: X \rightarrow B$ be a simple wrinkled fibration and let $\beta_{w}$ be the associated simplified broken Lefschetz fibration. Given a reference system $\mathcal{R}=\left\{R_{i}\right\}$ for $w$ with associated surface diagram $(\Sigma, \Gamma)$ there is a canonical Hurwitz system for $\beta_{w}$. Since the unsinking homotopy is supported near the cusps we can assume that the nothing happens around the reference paths. Now observe that the $\operatorname{arcs} R_{i}$ cut the higher-genus region into triangles each containing a single Lefschetz singularity of $\beta_{w}$. Thus, up to isotopy, there is a unique arc $L_{i}$ in the triangle bounded by $R_{i}$ and $R_{i+1}$ going from the reference fiber to the Lefschetz singularity and for the round singularity we take the arc $R=R_{1}$. According to Lekili [2009], the vanishing cycles of $\beta_{w}$ with respect to this Hurwitz system are given by

$$
\lambda_{i}=\tau_{c_{i}}\left(c_{i+1}\right) \text { and } \rho=c_{1} .
$$

We can go from the handle decomposition induced by $\beta_{w}$ to the one induced by $w$ using the following handlebody interpretation of the (un)sinking deformation.

Assume that we have a Lefschetz singularity next to a fold arc that is sinkable, that is, the Lefschetz and fold vanishing cycles intersect in one point. (In other words, it is the resulting of unsinking a cusp.) In terms of handle decompositions the situation before and after the sinking process is locally described in Figure 16. (These handle decompositions have already appeared in a disguised form in [Lekili 2009].) Clearly, both pictures describe a 4-ball and they are related by an obvious 

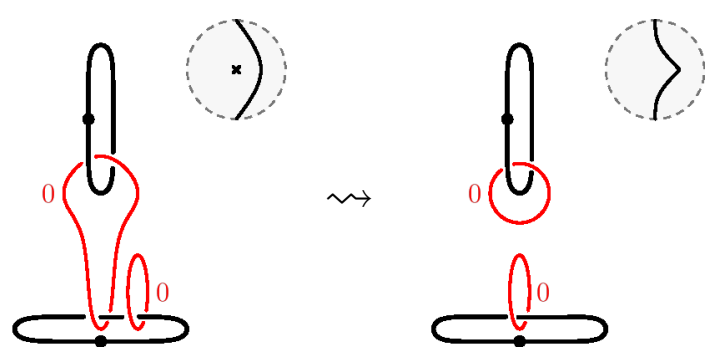

Figure 16. A Lefschetz singularity before and after sinking. (The Lefschetz 2-handle on the left runs over both 1-handles. One readily checks that it is correctly framed.)

2-handle slide. Indeed, to go from (a) to (b) one has to slide the Lefschetz handle over the fold handle in such a way that it unlinks from the lower 1-handle. Note that his handle slide is compatible with the fibration structures in the sense that the attaching curves stay on the fibers. Moreover, it mysteriously adjusts the framings exactly as needed.

Remark 4.15. Although the handle slide described above seems to be a correct interpretation of Lekili's (un)sinking deformation it is a priori not obvious why this should be true. In fact, the deformation is a combination of wrinkling, merging and flipping (see [Lekili 2009, Figure 8]) and does not seem very atomic. On the other hand, the handle slide is an atomic modification of the handlebodies. It would be interesting to see a 1-parameter family of Morse functions associated with the (un)sinking deformation that would exhibit the handle slide.

This shows that, if we start we the handle decomposition of $\beta_{w}$, then sliding $\lambda_{1}$ over $\rho=c_{1}$ produces a fiber framed attaching curve $\lambda_{1}^{\prime}$ which is isotopic to $c_{2}$. Successively sliding $\lambda_{i}$ over $\lambda_{i-1}^{\prime} \sim c_{i}$ results in fiber framed attaching curves $\lambda_{i}^{\prime}$ isotopic to $c_{i+1}$. Altogether we end up with fiber framed curves $\lambda_{1}^{\prime}, \ldots, \lambda_{l}^{\prime}, \rho$. The final observation is that $\lambda_{l}^{\prime}$ is isotopic to $\rho=c_{1}$ and can be unlinked and isolated from the rest of the diagram to form a zero framed unknot which cancels the 3-handle coming from the round singularity. What we are left with is the decomposition associated to $w$.

\section{Substitutions}

Let $\mathfrak{S}=(\Sigma, \Gamma)$ be a surface diagram and let $\Lambda$ be a subcircuit of $\Gamma$. If $\Lambda^{\prime}$ is any circuit that starts and ends with the same curves as $\Lambda$, then we can build a new surface diagram $\left(\Sigma, \Gamma^{\prime}\right)$ where $\Gamma^{\prime}$ is obtained by replacing $\Lambda$ with $\Lambda^{\prime}$. We call this operation a substitution of type $\left(\Lambda \mid \Lambda^{\prime}\right)$. Similar substitution techniques for Lefschetz fibrations are studied in [Endo and Gurtas 2010; Endo et al. 2011]. 
Passing to the associated simple wrinkled fibrations one can ask how such a substitution affects the total spaces. In the following we treat two instances in which this question can be answered. Our main tools are the handle decompositions exhibited in the previous section.

Let $Z$ be a compact 4-manifold, possibly with nonempty boundary. Recall that the operations of taking connected sums with $\overline{\mathbb{C} P^{2}}$ and $\Sigma^{2} \times S^{2}$ (taken in the interior of $Z$ ) are commonly known as blow-up and sum stabilization. We will be slightly more general and also call connected sums with $\mathbb{C} P^{2}$ blow-ups and connected sums with $\mathbb{C} P^{2} \# \overline{\mathbb{C} P^{2}}$, the twisted $S^{2}$-bundle over $S^{2}$, sum stabilizations. For convenience, we let

$$
\mathbb{S}_{k}:= \begin{cases}S^{2} \times S^{2} & \text { for } k \text { even } \\ \mathbb{C} P^{2} \# \overline{\mathbb{C} P^{2}} & \text { for } k \text { odd }\end{cases}
$$

and note that $\mathbb{S}_{k}$ is described by the $(0, k)$-framed Hopf link.

Lemma 5.1 (blow-ups and sum stabilizations). Let $\mathfrak{S}=(\Sigma, \Gamma)$ be a surface diagram and let $\mathfrak{S}^{\prime}$ be obtained from $\mathfrak{S}$ by a substitution of type

$$
\left(a, b \mid a, \tau_{b}^{ \pm 1}(a), b\right) .
$$

Furthermore, let $\mathfrak{S}^{\prime \prime}$ be obtained by a substitution of type

$$
\left(a, b \mid a, b, \tau_{b}^{k}(a), b\right) .
$$

Then $Z_{\mathfrak{S}^{\prime}}$ is diffeomorphic to the blow-up $Z_{\mathfrak{S}} \# \mp \mathbb{C} P^{2}$ and $Z_{\mathfrak{S}^{\prime \prime}}$ is diffeomorphic to the sum stabilization $Z_{\mathfrak{S}} \# \mathbb{S}_{-k}$.

Of course, any substitution is reversible so that whenever a surface diagram contains a configuration of the form $\left(a, \tau_{b}^{ \pm 1}(a), b\right)$ or $\left(a, b, \tau_{b}^{k}(a), b\right)$ the associated 4-manifold must be a blow-up or sum stabilization, respectively. We will call these blow-up (respectively sum stabilization) configurations.

Proof. By switching we can assume that $\Gamma=(\ldots, a, b)$, so $\Gamma^{\prime}\left(\ldots, a, \tau_{b}^{ \pm 1}(a), b\right)$ and $\Gamma^{\prime \prime}=\left(\ldots, a, b, \tau_{b}^{k}(a), b\right)$. Figure 17 shows the relevant parts of the handle decompositions of the associated 4-manifolds. The shaded ribbons indicate the regions that contain all the other fold handles. Note that the curves $a$ and $b$ appear as 0 -framed meridians to the dotted circles.

In the case of $Z_{\mathfrak{S}^{\prime}}$ we can use the meridians to unlink the curve corresponding to $\tau_{b}^{ \pm}(a)$ resulting in an unknot with framing $\mp 1$ which is isolated from the rest of the diagram. Furthermore, the rest of the diagram agrees with the diagram for $Z_{\mathfrak{S}}$ and the claim follows.

The argument for $Z_{\mathfrak{S}^{\prime \prime}}$ is almost the same. Again, by sliding over the meridians we can isolate the curves corresponding to $b$ and $\tau_{b}^{k}(a)$ from the rest of the diagram. This time we obtain a $(0,-k)$-framed Hopf link that represents a copy of $\mathbb{S}_{-k}$. 

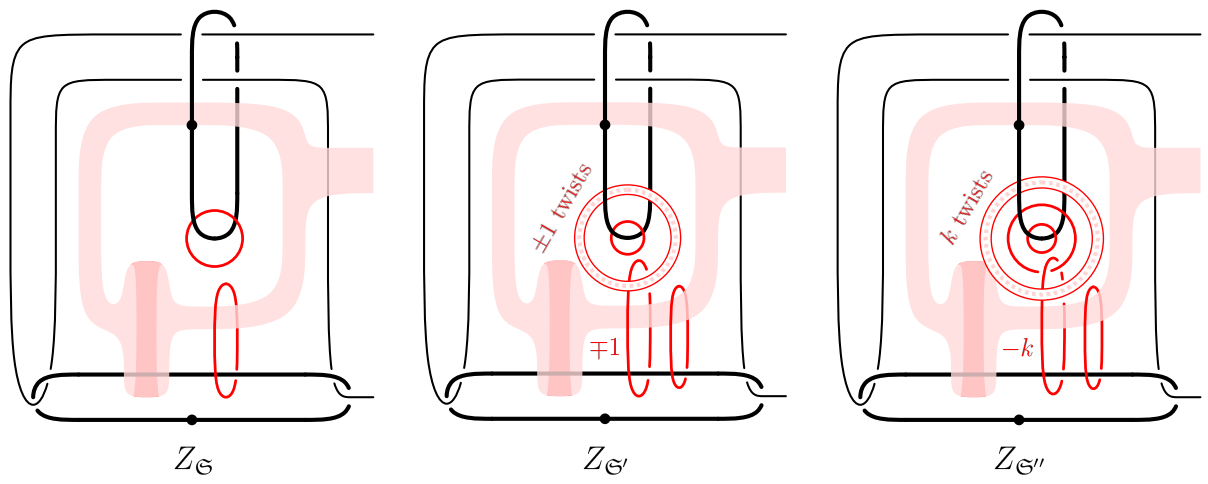

Figure 17. The relevant parts of the handle decompositions of $Z_{\mathfrak{S}}$, $Z_{\mathfrak{S}^{\prime}}$ and $Z_{\mathfrak{S}^{\prime \prime}}$. All 2-handles without framing coefficient are 0framed.

Proposition 5.2. Let $\mathfrak{S}, \mathfrak{S}^{\prime}$ and $\mathfrak{S}^{\prime \prime}$ be as in Lemma 5.1.

(1) All three diagrams have the same monodromy.

(2) If $\mathfrak{S}$ has trivial monodromy so that $Z_{\mathfrak{S}}$ closes off to a closed 4-manifold $X$, then $Z_{\mathfrak{S}^{\prime}}$ closes off to $X \# \mp \mathbb{C} P^{2}$ and $Z_{\mathfrak{S}^{\prime \prime}}$ closes off to $X \# \mathbb{S}_{k}$.

(3) Any closed 4-manifold obtained from $\mathfrak{S}^{\prime}$ (resp. $\mathfrak{S}^{\prime \prime}$ ) is a blow-up (resp. sum stabilization) of a manifold obtained from $\mathfrak{S}$.

Proof. The first statement follows directly from Lemma 5.1 since connected sums with closed manifold (taken in the interior) do not change the boundary.

For the other statements, observe that if one knows how to apply the method from Remark 4.12 for $\mathfrak{S}$, then one also knows it for $\mathfrak{S}^{\prime}$ and $\mathfrak{S}^{\prime \prime}$, and vice versa.

Another instance where a substitution corresponds to a well known cut-and-paste operation was observed in [Hayano 2012, Lemma 6.13]. Assume that a surface diagram $\mathfrak{S}$ contains a curve $c \subset \Sigma$. If $d \subset \Sigma$ is geometrically dual to $c$, then one can perform a substitution of type $(c \mid c, d, c)$ and Hayano shows that if $\mathfrak{S}^{\prime}$ denotes the resulting surface diagram, then $Z_{\mathfrak{S}^{\prime}}$ is obtained from $Z_{\mathfrak{S}}$ by a surgery on the curve $\delta \subset \Sigma \subset Z_{\mathfrak{S}}$ with respect to its fiber framing, that is, the framing induced by the its canonical framing in $\Sigma$ together with the framing of $\Sigma$ in $Z_{\mathfrak{S}}$ as a regular fiber of $w_{\mathfrak{S}}: Z_{\mathfrak{S}} \rightarrow D^{2}$.

One immediately notices that our sum-stabilization substitution is a special case of this construction. However, it also paves the way for the following minor generalization of the surgery substitution which captures not only the fiber framed surgery but also the one with the opposite framing. (Recall that an embedded circle in an orientable 4-manifold always has trivial normal bundle and there are exactly two framings, since $\pi_{1}(\mathrm{SO}(3)) \cong \mathbb{Z}_{2}$.) 

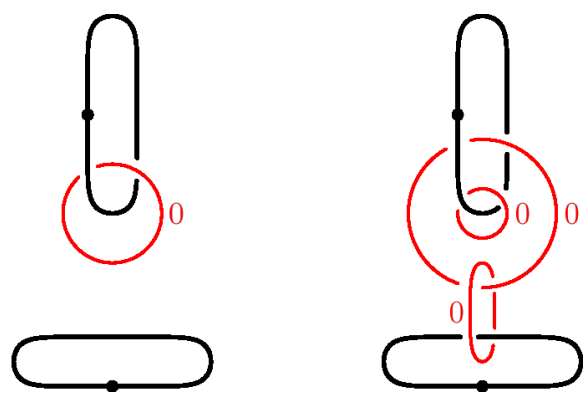

Figure 18. Hayano's surgery substitution: neighborhoods with vanishing cycle $c$ (left) and vanishing cycles $c, d, c$ (right).

Lemma 5.3. Let $\mathfrak{S}$ and $\mathfrak{S}^{\prime}$ be two surface diagrams with the same underlying surface $\Sigma$ and let $c, d \subset \Sigma$ be a geometrically dual pair of simple closed curves. If $\mathfrak{S}^{\prime}$ is obtained from $\mathfrak{S}$ by a substitution of type $\left(c \mid c, \tau_{c}^{k}(d), c\right)$, then $Z_{\mathfrak{S}^{\prime}}$ is obtained from $Z_{\mathfrak{S}}$ by a surgery on $d \subset \Sigma \subset X$ with respect to the fiber framing when $k$ is even and the opposite framing when $k$ is odd.

Proof. As in Hayano's proof, it is enough to work in a neighborhood of $c \cup d$ which we can assume to be a punctured torus. Using our handle decomposition instead of the ones from broken Lefschetz fibrations, the effect of Hayano's surgery substitution, that is, the case when $k=0$, looks as in Figure 18, where $c$ (respectively $d$ ) appears as the meridian of the upper (respectively lower) 1-handle. To obtain the other even cases, observe that in Figure 18, right, we can slide the 2-handle corresponding to $d$ once over each 2-handle corresponding to $c$ in the same direction. Depending on the direction this changes the framing coefficient by \pm 2 and one readily checks that the resulting diagram shows a neighborhood with vanishing cycles $\left(c, \tau_{c}^{\mp 2}(d), c\right)$. Repeating this trick one can obtain all configurations with even $k$ and they will all describe the fiber framed surgery on $d$.

As shown in [Gompf and Stipsicz 1999, Example 8.4.6] the surgery with the opposite framing can be realized by inserting a pair of a Lefschetz vanishing cycle and an achiral Lefschetz vanishing cycle which are both parallel to $d$. But Figure 19 shows that the result is the same as a substitution of type $\left(c \mid c, \tau_{c}^{-1}(d), c\right)$ which corresponds to $k=-1$. Moreover, the arguments for shifting the value of $k$ by multiples of 2 works just as in the fiber framed case.

Using Lemma 5.3, the sum stabilization can be interpreted as performing surgery on a null-homotopic curve with either of its framing. Indeed, as $d$ one takes one of the adjacent vanishing cycles of $c$ in $\mathfrak{S}$ which is clearly null-homotopic in $Z_{\mathfrak{S}}$.

It would be interesting to interpret other cut-and-paste operations on 4-manifolds as substitutions in surface diagrams. For example, it is reasonable to expect such 


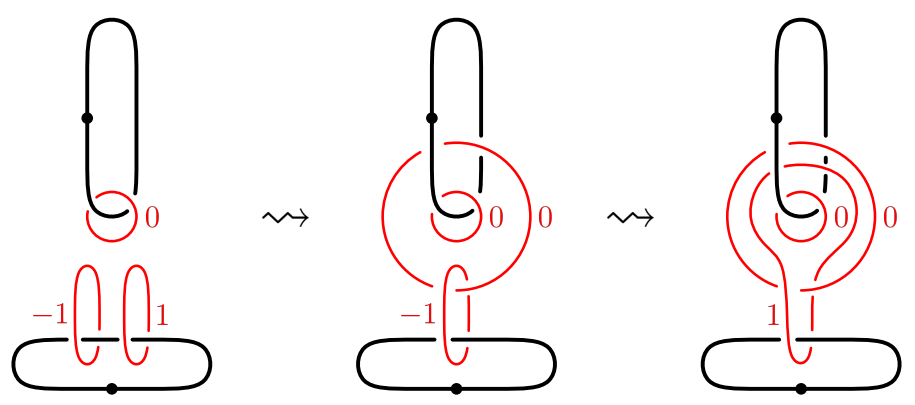

Figure 19. Surgery with the opposite framing.

an interpretation for certain rational blow downs which can be described in terms of Lefschetz fibrations; see [Endo et al. 2011]. However, we settle for blow-ups and sum stabilizations in this paper.

\section{Manifolds with genus-one simple wrinkled fibrations}

In this section we prove Theorem 1.2. Our strategy is to use Proposition 5.2 to construct some genus-one simple wrinkled fibrations and then show that this construction gives all such fibrations.

We begin with the construction of genus-one simple wrinkled fibrations over $S^{2}$. As before, we denote by $\mathbb{S}_{k}$ the closed 4-manifolds described by the $(0, k)$-framed Hopf link and we define a family of manifolds

$$
X_{k l m n}=\mathbb{S}_{k} \# l\left(S^{2} \times S^{2}\right) \# m \mathbb{C} P^{2} \# n \overline{\mathbb{C} P^{2}}, \quad k \in\{0,1\}, \quad l, m, n \geq 0 .
$$

Note that these are precisely the manifolds in Theorem 1.2. Recall that $\mathbb{S}_{k}$ is an $S^{2}$ bundle over $S^{2}$. By performing a birth on a suitable bundle projection $\mathbb{S}_{k} \rightarrow S^{2}$ we obtain a simple wrinkled fibration with two cusps. We can then use Proposition 5.2 to add the other summands at will. Thus, in order to prove Theorem 1.2, it remains to show the following.

Proposition 6.1. Let $w: X \rightarrow S^{2}$ be a simple wrinkled fibration of genus one. Then $X$ is diffeomorphic to some $X_{k l m n}$ described in (6-1).

Remark 6.2. The reason for our small reformulation of Theorem 1.2 is that, while the original formulation is cleaner, the new one is more in tune with the structure of the proof.

The key to the proof of Proposition 6.1 is the simple nature of simple closed curves on the torus. Indeed, the two facts that two oriented simple closed curves on the torus are isotopic if and only if they are homologous and that the (absolute value of the) algebraic and geometric intersection numbers agree allow us to transfer the whole 
discussion of genus 1 surface diagrams into the homology group $H_{1}\left(T^{2}\right) \cong \mathbb{Z} \oplus \mathbb{Z}$ simply by choosing orientations on the curves. Building on this observation we obtain the following result about the structure of genus-one surface diagrams.

Lemma 6.3. Any closed circuit on the torus of length at least three contains blow-up or sum stabilization configurations (as described in Lemma 5.1).

Proof. Let $\Gamma=\left(c_{1}, \ldots, c_{l}\right)$ be a (not necessarily closed) circuit on the torus of length $c \geq 3$. As usual, we choose an arbitrary orientation on $c_{1}$ and orient the remaining curves by requiring that $\left\langle c_{i}, c_{i+1}\right\rangle=1$ for $i<l$ so that we can consider each $c_{i}$ as an element of $H_{1}\left(T^{2}\right)$.

We first observe that, since any two adjacent curves in $\Gamma$ are algebraically dual, they form a basis of $H_{1}\left(T^{2}\right)$. In particular, for $i \geq 3$ we can write

$$
c_{i}=k_{i} c_{i-1}-c_{i-2}, \quad k_{i} \in \mathbb{Z}
$$

where the coefficient of $c_{i-2}$ determined by our convention that $\left\langle c_{i-1}, c_{i}\right\rangle=1$. This shows that if we denote by $\sigma_{i}:=\left\langle c_{1}, c_{i}\right\rangle$ the algebraic intersection number between $c_{1}$ and $c_{i}$, then we obtain a recursive formula

$$
\sigma_{i}=k_{i} \sigma_{i-1}-\sigma_{i-2}
$$

for $i \geq 3$ with initial values $\sigma_{1}=0$ and $\sigma_{2}=1$. At this point we note that $\Gamma$ is closed if and only if $\left|\sigma_{l}\right|=1$.

We claim that if $\left|k_{i}\right| \geq 2$ for all $i \geq 3$, then $\left|\sigma_{i+1}\right|>\left|\sigma_{i}\right|$ for all $i$. This follows inductively since $\left|\sigma_{2}\right|>\left|\sigma_{1}\right|$ and from (6-2) we get

$$
\left|\sigma_{i+1}\right|=\left|k_{i+1} \sigma_{i}-\sigma_{i-1}\right| \geq|| k_{i+1}|| \sigma_{i}|-| \sigma_{i-1}||=\left|k_{i+1}\right|\left|\sigma_{i}\right|-\left|\sigma_{i-1}\right|>\left|\sigma_{i}\right|,
$$

where we have used the reverse triangle inequality, the induction hypothesis and the assumption that $\left|k_{i+1}\right| \geq 2$. As a consequence, we see that if $\Gamma$ is closed, then we must have $\left|k_{i}\right| \leq 1$ for some $i \geq 3$.

Assume first that $k_{i}= \pm 1$. To keep the notation transparent we momentarily rename the relevant curves to

$$
\left(c_{i-2}, c_{i-1}, c_{i}\right)=:(a, \xi, b) .
$$

By assumption, $b= \pm \xi-a$ and thus $\xi= \pm(a+b)$ and the orientation convention shows that $\langle a, b\rangle= \pm 1$. By invoking the Picard-Lefschetz formula (Proposition 2.7) we obtain

$$
\tau_{a}^{ \pm 1}(b)=b \pm\langle a, b\rangle a=a+b= \pm \xi,
$$

which, after forgetting the orientations again, reveals the excerpt of $\Gamma$ shown in (6-3) as a blow-up configuration.

A similar argument exhibits a sum-stabilization configuration in the remaining case when $k_{i}=0$. The details are left to the reader. 
The proof of Proposition 6.1, and thus of Theorem 1.2, is now very easy.

Proof of Proposition 6.1. Any genus-one simple wrinkled fibration over $S^{2}$ is obtained by closing off a manifold $Z_{\mathfrak{S}}$ associated to a surface diagram $\mathfrak{S}=\left(T^{2}, \Gamma\right)$. Moreover, any such diagram $\mathfrak{S}$ can be closed off since the mapping class group of the lower-genus fiber is trivial. By Lemma 6.3 and Proposition 5.2(3) we can successively split off summands of the form $\pm \mathbb{C} P^{2}$ and $\mathbb{S}_{k}$ until the remaining surface diagram, say $\mathfrak{S}_{0}$ has a circuit of length two. It is easy to see that $Z_{\mathfrak{S}_{0}}$ is the trivial disk bundle $S^{2} \times D^{2}$. (Either by drawing a Kirby diagram or by observing that any simple wrinkled fibration with two cusps is homotopic to a bundle projection.) Thus there are exactly two ways to close off the fibration, producing a summand of the form $\mathbb{S}_{0} \cong S^{2} \times S^{2}$ or $\mathbb{S}_{1} \cong \mathbb{C} P^{2} \# \overline{\mathbb{C} P^{2}}$.

\section{Concluding remarks}

The theory of simple wrinkled fibrations and surface diagrams is still in a very early stage and at this point it raises more questions then it provides answers. We would like to point out what we consider as some of the major problems in the subject as well as to indicate some further developments.

7A. Closed 4-manifolds. The ultimate goal is to use surface diagrams to study closed 4-manifolds. Unfortunately, it turns out that most surface diagrams do not describe closed manifolds since they have nontrivial monodromy and it is usually a hard problem to determine whether a given surface diagram has trivial monodromy. The following is thus of great interest.

Problem 7.1. Find at least necessary conditions for a surface diagram to have trivial monodromy that are easier to check.

The next major problem was already mentioned on page 292. If a surface diagram of sufficiently high genus is known to have trivial monodromy, then it determines a unique closed 4-manifold together with a simple wrinkled fibration over $S^{2}$ by closing off the associated fibration over the disk. However, for practical purposes the information on how to close off is encoded too implicitly in the surface diagram. For example, by simply looking at the surface diagram it not at all clear how to answer the following very reasonable questions about the corresponding simple wrinkled fibration over $S^{2}$ :

- Does the fibration have a section?

- What can be said about the homology class of the fiber? Is it trivial, primitive, torsion, ....?

- What is the fundamental group, homology, etc. of the total space? 
What is missing is one more piece of information which is roughly the (framed) attaching curve of the last 2-handle. One can also reformulate this issue in terms of mapping class groups (see [Hayano 2012], for example).

Problem 7.2. Find a practical method to determine the missing piece of information from a surface diagram with trivial monodromy.

7B. Higher-genus fibrations. The fact that any (achiral) Lefschetz fibration can be turned into a simple wrinkled fibration of one genus higher suggests the philosophy that simple wrinkled fibrations of a fixed genus might behave similarly as (achiral) Lefschetz fibrations of one genus lower.

This analogy works rather well for the lowest possible fiber genera. Indeed, our result about genus-one simple wrinkled fibrations looks very similar to the (rather trivial) classification of genus zero (achiral) Lefschetz fibrations, the latter being blow-ups of either $S^{2} \times S^{2}$ or $\mathbb{C} P^{2} \# \overline{\mathbb{C} P^{2}}$.

Following this train of thought one might hope to be able to say something useful about the classification of genus two simple wrinkled fibrations over $S^{2}$ but one should expect to be lost as soon as the genus is three or higher. However, it is nonetheless conceivable that part of the classification scheme that works in the genus-one case might carry over to higher-genus fibrations, as we will now explain.

Let $\mathfrak{S}=\left(\Sigma ; c_{1}, \ldots, c_{l}\right)$ be a surface diagram and assume that for some $2<k<l$ the curve $c_{k}$ is geometrically dual to $c_{1}$. Then there is an obvious way to decompose $\mathfrak{S}$ into the two smaller surface diagrams $\left(\Sigma ; c_{1}, \ldots, c_{k}\right)$ and $\left(\Sigma ; c_{1}, c_{k}, \ldots, c_{l}\right)$. Repeating this process we eventually obtain a decomposition of $\mathfrak{S}$ into a collection of surface diagrams with the property that no pair of nonadjacent curves has geometric intersection number one. Let us call such a surface diagram irreducible.

In terms of the simple wrinkled fibration associated to $\mathfrak{S}$ the above decomposition of $\mathfrak{S}$ should correspond to merging the fold arcs that induce $c_{1}$ and $c_{k}$. (As shown in [Lekili 2009], the necessary and sufficient condition for a fold merge is exactly that the vanishing cycles of the fold arcs are geometrically dual.) The result is a wrinkled fibration that naturally decomposes as a boundary fiber sum of the two simple wrinkled fibrations associated to the parts of the decomposition of $\mathfrak{S}$.

This suggests that any descending simple wrinkled fibration over the disk naturally decomposes into a boundary fiber sum of irreducible fibrations where we call a simple wrinkled fibration irreducible if its surface diagram is irreducible. Consequently, the classification of descending simple wrinkled fibrations splits into two parts: the classification of irreducible fibrations and understanding the effect of boundary fiber sums.

The genus-one classification fits into this scheme as follows. Our arguments show that the only irreducible surface diagrams of genus-one are given by the blow-up configurations $\left(a, \tau_{a}^{ \pm 1}(b), b\right)$ and the sum-stabilization configurations 
$\left(a, b, \tau_{b}^{k}(a), b\right)$ for $k \neq 1$. Using the handle decompositions it is easy to identify the corresponding manifolds. (They are the connected sum of $S^{2} \times D^{2}$ with either $\pm \mathbb{C} P^{2}, S^{2} \times S^{2}$ or $\mathbb{C} P^{2} \# \overline{\mathbb{C} P^{2}}$.) Furthermore, the boundary fiber sums are performed along spheres and are thus easy to understand.

Making these arguments precise requires an understanding of the effect of merging folds and cusps on surface diagrams.

7C. Uniqueness of surface diagrams. Given the fact that all closed 4-manifolds can be described by surface diagrams, it is natural to ask for a set of moves to relate different surface diagrams that describe the same manifold, similar to the situation of 3-manifolds and Heegaard diagrams.

A first step in this direction was taken by Williams [2011] who relates the surface diagrams of homotopic simple wrinkled fibrations over $S^{2}$ of genus at least three. He shows that any two homotopic simple wrinkled fibrations can be connected by a special homotopy that is made up of four basic building blocks. These building blocks are simple enough to understand their effect on the initial surface diagram (see also [Hayano 2012]).

So far this is completely analogous to the 3 -dimensional context. A new phenomenon in the 4-dimensional context is that two simple wrinkled fibrations on a given 4-manifold are not necessarily homotopic. The structure of the set $\pi^{2}(X):=\left[X, S^{2}\right]$ of homotopy classes of maps from a closed 4-manifold to the 2-sphere - also known as the second cohomotopy set of $X$ - is described in [Kirby et al. 2012] (see also the references therein). Our results show that an equivalence class of surface diagrams for $X$ determines an orbit of the action of the diffeomorphism group of $X$ on $\pi^{2}(X)$. This action is usually neither trivial, as shown by the two projections of $S^{2} \times S^{2}$ which are interchanged by flipping the factors, nor transitive since the action of the diffeomorphism group on the second homology group preserves divisibility. Thus, reparametrizing a surface diagram can change the homotopy class of its simple wrinkled fibration but one cannot expect to obtain all homotopy classes in this way.

A general method for relating broken fibrations in different homotopy classes is the projection move mentioned in [Williams 2010] but it is not at all obvious how to interpret this procedure in terms of surface diagrams. Altogether, the problem of relating surface diagram with nonhomotopic fibrations is still wide open.

\section{Acknowledgements}

This work is part of the author's ongoing PhD project carried out at the Max Planck Institute for Mathematics in Bonn, Germany. The author would like to thank his advisor Prof. Dr. Peter Teichner for his support as well as İnanç Baykur for helpful comments on an early draft of this paper. The author is supported by an IMPRS Scholarship of the Max Planck Society. 


\section{References}

[Akbulut and Karakurt 2008] S. Akbulut and Ç. Karakurt, "Every 4-manifold is BLF", J. Gökova Geom. Topol. GGT 2 (2008), 83-106. MR 2009k:57037 Zbl 1209.57015

[Auroux et al. 2005] D. Auroux, S. K. Donaldson, and L. Katzarkov, "Singular Lefschetz pencils", Geom. Topol. 9 (2005), 1043-1114. MR 2005m:53164 Zbl 1077.53069

[Baykur 2008] R. İ. Baykur, "Existence of broken Lefschetz fibrations", Int. Math. Res. Not. 2008 (2008), Art. ID rnn 101, 15. MR 2010b:57026 Zbl 1179.57011

[Baykur 2009] R. İ. Baykur, "Topology of broken Lefschetz fibrations and near-symplectic fourmanifolds", Pacific J. Math. 240:2 (2009), 201-230. MR 2010c:57035 Zbl 1162.57011

[Baykur and Kamada 2010] R. I. Baykur and S. Kamada, "Classification of broken Lefschetz fibrations with small fiber genera", preprint, 2010. arXiv 1010.5814v2

[Boardman 1967] J. M. Boardman, "Singularities of differentiable maps", Inst. Hautes Études Sci. Publ. Math. 33 (1967), 21-57. MR 37 \#6945 Zbl 0165.56803

[Donaldson 1999] S. K. Donaldson, "Lefschetz pencils on symplectic manifolds", J. Differential Geom. 53:2 (1999), 205-236. MR 2002g:53154 Zbl 1040.53094

[Earle and Eells 1969] C. J. Earle and J. Eells, "A fibre bundle description of Teichmüller theory", J. Differential Geometry 3 (1969), 19-43. MR 43 \#2737a Zbl 0185.32901

[Endo and Gurtas 2010] H. Endo and Y. Z. Gurtas, "Lantern relations and rational blowdowns", Proc. Amer. Math. Soc. 138:3 (2010), 1131-1142. MR 2011a:57047 Zbl 1198.57017

[Endo et al. 2011] H. Endo, T. E. Mark, and J. Van Horn-Morris, "Monodromy substitutions and rational blowdowns”, J. Topol. 4:1 (2011), 227-253. MR 2012b:57051 Zbl 1234.57037

[Farb and Margalit 2011] B. Farb and D. Margalit, A primer on mapping class groups, Princeton Mathematical Series 49, Princeton University Press, 2011. MR 2012h:57032 Zbl 1245.57002

[Gay and Kirby 2007] D. T. Gay and R. Kirby, "Constructing Lefschetz-type fibrations on fourmanifolds”, Geom. Topol. 11 (2007), 2075-2115. MR 2009b:57048 Zbl 1135.57009

[Gay and Kirby 2011a] D. T. Gay and R. Kirby, "Indefinite Morse 2-functions; broken fibrations and generalizations", preprint, 2011. arXiv 1102.0750v2

[Gay and Kirby 2011b] D. T. Gay and R. C. Kirby, "Fiber-connected, indefinite Morse 2-functions on connected n-manifolds", Proc. Natl. Acad. Sci. USA 108:20 (2011), 8122-8125. MR 2012k:57041

[Gay and Kirby 2012] D. T. Gay and R. Kirby, "Reconstructing 4-manifolds from Morse 2-functions", preprint, 2012. Zbl 06124834 arXiv 1202.3487

[Golubitsky and Guillemin 1973] M. Golubitsky and V. Guillemin, Stable mappings and their singularities, Graduate Texts in Mathematics 14, Springer, New York, 1973. MR 49 \#6269 Zbl 0294.58004

[Gompf and Stipsicz 1999] R. E. Gompf and A. I. Stipsicz, 4-manifolds and Kirby calculus, Graduate Studies in Mathematics 20, American Mathematical Society, Providence, RI, 1999. MR 2000h:57038 Zbl 0933.57020

[Hayano 2011] K. Hayano, "On genus-1 simplified broken Lefschetz fibrations", Algebr. Geom. Topol. 11:3 (2011), 1267-1322. MR 2012f:57067 Zbl 1229.57017

[Hayano 2012] K. Hayano, "Modification rule of monodromies in $R_{2}$-move", preprint, 2012. arXiv 1203.4299v1

[Ivanov 1992] N. V. Ivanov, Subgroups of Teichmüller modular groups, Translations of Mathematical Monographs 115, American Mathematical Society, Providence, RI, 1992. MR 93k:57031 Zbl 0776.57001 
[Kas 1980] A. Kas, "On the handlebody decomposition associated to a Lefschetz fibration", Pacific J. Math. 89:1 (1980), 89-104. MR 82f:57012 Zbl 0457.14011

[Kirby et al. 2012] R. Kirby, P. Melvin, and P. Teichner, "Cohomotopy sets of 4-manifolds", preprint, 2012. arXiv 1203.1608v1

[Lekili 2009] Y. Lekili, "Wrinkled fibrations on near-symplectic manifolds", Geom. Topol. 13:1 (2009), 277-318. MR 2009k:57043 Zbl 1164.57006

[Levine 1964] H. I. Levine, "The singularities, $S_{1} q "$, Illinois J. Math. 8 (1964), 152-168. MR 28 \#2560 Zbl 0124.38801

[Morin 1965] B. Morin, "Formes canoniques des singularités d'une application différentiable", $C . R$. Acad. Sci. Paris 260 (1965), 5662-5665. MR 31 \#5212 Zbl 0178.26801

[Williams 2010] J. Williams, "The $h$-principle for broken Lefschetz fibrations", Geom. Topol. 14:2 (2010), 1015-1061. MR 2011d:57066 Zbl 1204.57027

[Williams 2011] J. D. Williams, "Topology of surface diagrams of smooth 4-manifolds", Proc. Natl. Acad. Sci. USA 108:20 (2011), 8126-8130. MR 2012f:57066

Received August 3, 2012. Revised October 12, 2012.

STEFAN BEHRENS

Max Planck Institute For Mathematics

VIVATSGASSE 7

D-53111 BONN

GERMANY

sbehrens@mpim-bonn.mpg.de 


\title{
PACIFIC JOURNAL OF MATHEMATICS
}

\author{
msp.org/pjm
}

Founded in 1951 by E. F. Beckenbach (1906-1982) and F. Wolf (1904-1989)

\section{EDITORS}

V. S. Varadarajan (Managing Editor)

Department of Mathematics

University of California

Los Angeles, CA 90095-1555

pacific@math.ucla.edu

Paul Balmer

Department of Mathematics

University of California

Los Angeles, CA 90095-1555

balmer@math.ucla.edu

Daryl Cooper

Department of Mathematics

University of California

Santa Barbara, CA 93106-3080 cooper@math.ucsb.edu

Jiang-Hua $\mathrm{Lu}$

Department of Mathematics

The University of Hong Kong

Pokfulam Rd., Hong Kong jhlu@maths.hku.hk
Don Blasius

Department of Mathematics University of California

Los Angeles, CA 90095-1555

blasius@math.ucla.edu

Robert Finn

Department of Mathematics Stanford University

Stanford, CA 94305-2125

finn@math.stanford.edu

Sorin Popa

Department of Mathematics

University of California

Los Angeles, CA 90095-1555

popa@math.ucla.edu

Paul Yang

Department of Mathematics

Princeton University

Princeton NJ 08544-1000

yang@math.princeton.edu

\section{PRODUCTION}

Silvio Levy, Scientific Editor, production@msp.org

\section{SUPPORTING INSTITUTIONS}

ACADEMIA SINICA, TAIPEI

CALIFORNIA INST. OF TECHNOLOGY

INST. DE MATEMÁTICA PURA E APLICADA

KEIO UNIVERSITY

MATH. SCIENCES RESEARCH INSTITUTE

NEW MEXICO STATE UNIV.

OREGON STATE UNIV.

\author{
STANFORD UNIVERSITY \\ UNIV. OF BRITISH COLUMBIA \\ UNIV. OF CALIFORNIA, BERKELEY \\ UNIV. OF CALIFORNIA, DAVIS \\ UNIV. OF CALIFORNIA, LOS ANGELES \\ UNIV. OF CALIFORNIA, RIVERSIDE \\ UNIV. OF CALIFORNIA, SAN DIEGO \\ UNIV. OF CALIF., SANTA BARBARA
}

\author{
Vyjayanthi Chari \\ Department of Mathematics \\ University of California \\ Riverside, CA 92521-0135 \\ chari@math.ucr.edu \\ Kefeng Liu \\ Department of Mathematics \\ University of California \\ Los Angeles, CA 90095-1555 \\ liu@math.ucla.edu \\ Jie Qing \\ Department of Mathematics \\ University of California \\ Santa Cruz, CA 95064 \\ qing@cats.ucsc.edu
}

These supporting institutions contribute to the cost of publication of this Journal, but they are not owners or publishers and have no responsibility for its contents or policies.

See inside back cover or msp.org/pjm for submission instructions.

The subscription price for 2013 is US \$400/year for the electronic version, and \$485/year for print and electronic.

Subscriptions, requests for back issues and changes of subscribers address should be sent to Pacific Journal of Mathematics, P.O. Box 4163, Berkeley, CA 94704-0163, U.S.A. The Pacific Journal of Mathematics is indexed by Mathematical Reviews, Zentralblatt MATH, PASCAL CNRS Index, Referativnyi Zhurnal, Current Mathematical Publications and the Science Citation Index.

The Pacific Journal of Mathematics (ISSN 0030-8730) at the University of California, c/o Department of Mathematics, 798 Evans Hall \#3840, Berkeley, CA 94720-3840, is published ten to twelve times a year. Periodical rate postage paid at Berkeley, CA 94704, and additional mailing offices. POSTMASTER: send address changes to Pacific Journal of Mathematics, P.O. Box 4163, Berkeley, CA 94704-0163.

PJM peer review and production are managed by EditFLOW ${ }^{\circledR}$ from Mathematical Sciences Publishers.

PUBLISHED BY 


\section{PACIFIC JOURNAL OF MATHEMATICS}

Volume $264 \quad$ No. $2 \quad$ August 2013

On 4-manifolds, folds and cusps

STEFAN BEHRENS

Thin $r$-neighborhoods of embedded geodesics with finite length and

307 negative Jacobi operator are strongly convex

PHILIPPE DELANOË

Eigenvalues of perturbed Laplace operators on compact manifolds

ASMA HASSANNEZHAD

Four equivalent versions of nonabelian gerbes

THOMAS NIKOLAUS and KONRAD WALDORF

On nonlinear nonhomogeneous resonant Dirichlet equations

421

Nikolaos S. PAPAGEORGIOU and GEORGE SMYRLIS

A geometric model of an arbitrary real closed field

455

STANISŁAW SPODZIEJA

Twisted K-theory for the orbifold $[* / G]$

471

MARIO VELÁSQUEZ, EdWARd BECERRA and HERMES

MARTINEZ

Linear restriction estimates for the wave equation with an inverse square potential

JUNYONG ZHANG and JIQIANG ZHENG 